\title{
Endoscopic treatment of benign biliary stricture using different stents: a systematic review and meta-analysis
}

\author{
Zun Fan, Xin Zhao, Renting Ji, Jiacheng Li, Yang Shi \\ Department of General Surgery, The First Affiliated Hospital of Soochow University, Suzhou, China
}

Videosurgery Miniinv 2022; 17 (1): 35-60

DOI: https://doi.org/10.5114/wiitm.2021.108418

\begin{abstract}
Introduction: Biodegradable biliary stents (BDBSs), fully covered self-expanded metal stents (FCSEMSs) and multiple plastic stents (MPSS) were common stents in endoscopic treatment of benign biliary stricture (BBS).

Aim: To evaluate the effectiveness of these 3 stents in BBS management.

Material and methods: The PubMed, Web of Science, Cochrane Library, and Wiley Library databases were searched for studies that provided data about BBS and stent therapy.

Results: We found that BDBSs were associated with the highest clinical success rate $(0.76,95 \% \mathrm{Cl}: 0.71-0.80)$, followed by MPSS (0.69, 95\% Cl: 0.63-0.74), and FCSEMSS (0.67, 95\% Cl: 0.63-0.71). BDBSs also had a relatively high probability of technical success, at 1.00 (95\% Cl: 1.00-1.00), superior to MPSS (0.95, 95\% Cl: 0.88-0.99) and FCSEMSS (0.90, 95\% Cl: 0.85-0.94). The treatment success rate for BDBSs (1.00, 95\% Cl: 1.00-1.00) was also higher than for MPSS $(0.88,95 \% \mathrm{Cl}: 0.72-0.98)$ and FCSMESS $(0.82,95 \% \mathrm{Cl}: 0.76-0.87)$. However, BDBSs had the highest stricture recurrence rate $(0.21,95 \% \mathrm{Cl}: 0.16-0.26)$, compared with FCSEMSs $(0.11,95 \% \mathrm{Cl}: 0.08-0.15)$ and MPSS (0.07, 95\% Cl: 0.03-0.13).
\end{abstract}

Conclusions: Patients with BBS are likely to receive a satisfied outcome when treated with BDBSs.

Key words: benign biliary stricture, stents, endoscopy, meta-analyses.

\section{Introduction}

Benign biliary stricture (BBS) is defined as any narrowing along the extrahepatic bile duct with less than $75 \%$ diameter of unaffected region [1]. Most cases result from the 2 leading pathogeneses: iatrogenic biliary injury and inflammation damage [2-4]. The condition of iatrogenic biliary injury includes open/laparoscopic cholecystectomy, duct-duct anastomosis after liver transplantation (LT), etc. [4-7]. Inflammation damage is mainly from chronic pancreatitis (CP) and primary sclerosing cholangitis [3, 4]. The incidence of BBS is about $1 \%$ for open cholecystectomy, $0.23-0.42 \%$ for laparoscopic cholecystectomy, 3-46\% for CP, 5-15\% for deceased LT, and
28-32\% for living-donor transplantation [8-12]. BBS may lead to elevation of serum bilirubin, impairment of liver function, and bacterial growth in the biliary tree. If BBS is not recognized in time and managed properly, these patients can suffer from even worse prognosis because of life-threatening complications such as secondary biliary cirrhosis, portal hypertension, and cholangitis [4, 13]. Therefore, every patient with BBS should receive aggressive treatment to release biliary obstruction effectively.

Currently, endoscopic intervention with stent implantation has been widely adopted for the treatment of BBS (Photo 1) [4]. With accelerated development of biomedical materials, endoscopic stents are continuously evolving, which includes multiple

Address for correspondence

Xin Zhao MD, PhD, Department of General Surgery, The First Affiliated Hospital of Soochow University, 188 Shizi St, Gusu District,

Suzhou 215006, Jiangsu Province, China, e-mail: zx15152881615@163.com; Yang Shi PhD, Department of General Surgery, The First Affiliated

Hospital of Soochow University, 188 Shizi St, Gusu District, Suzhou 215006, Jiangsu Province, China, e-mail: shiyang9962@163.com 

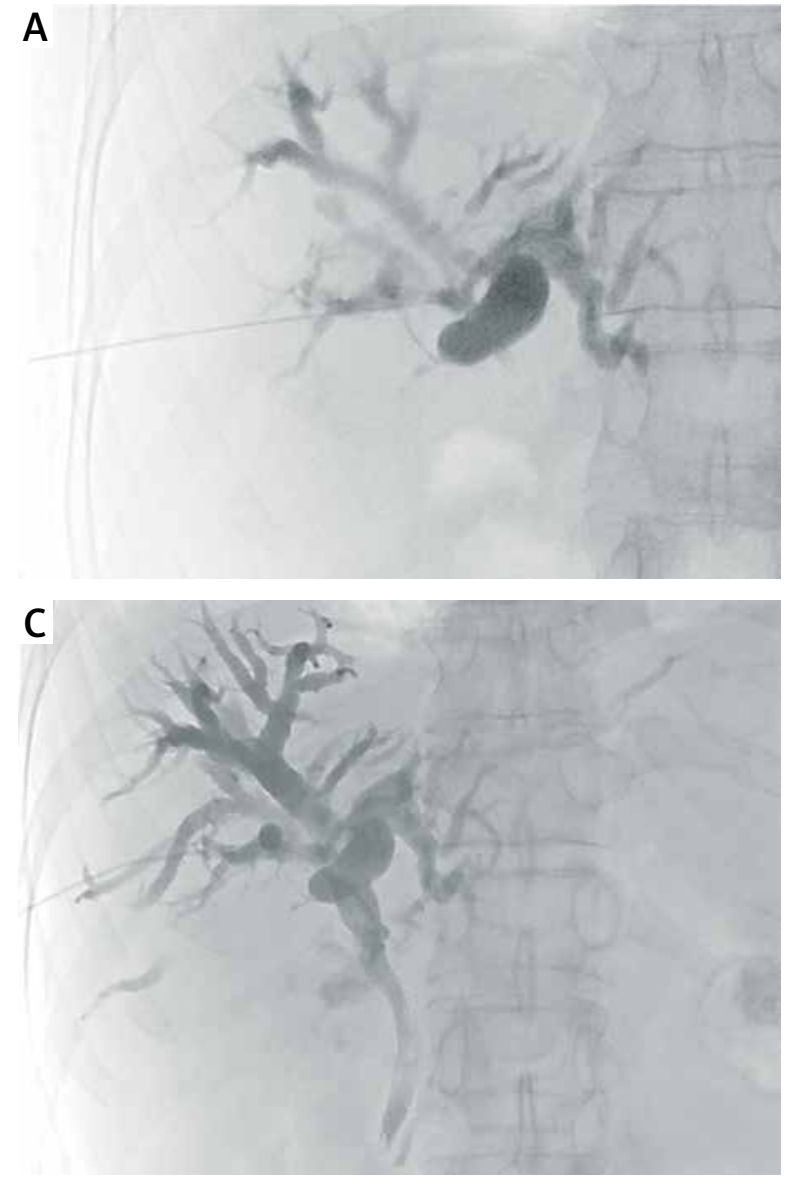

plastic stents (MPSs), fully covered self-expanded metal stents (FCSEMSs), and biodegradable biliary stents (BDBSs), et al. [4]. Among them, MPSs are the most used because of their cheap price, low technical requirements, and acceptable long-term results. However, this kind of stent requires repeated interventions to maintain its therapeutic effect, increasing the incidence of operation-related complications, such as pancreatitis, haemobilia, and abdominal pain [14]. FCSEMSs are another kind of stent with extensive applications. Previous studies have reported that a single FCSEMS is able to provide a radial dilation similar to that of three 10F plastic stents, and thus free from the trouble of frequent interventions [1]. The main drawback of FCSEMSs is frequent occurrence of stent migration (4-41\%), often resulting in treatment failure [6]. BDBSs are a relatively new kind of stent, and they are reported to have a similar radial expansion force with FCSEMSs. This kind of stent is biodegradable, and so does not need to be removed specially $[15,16]$. The main drawback of

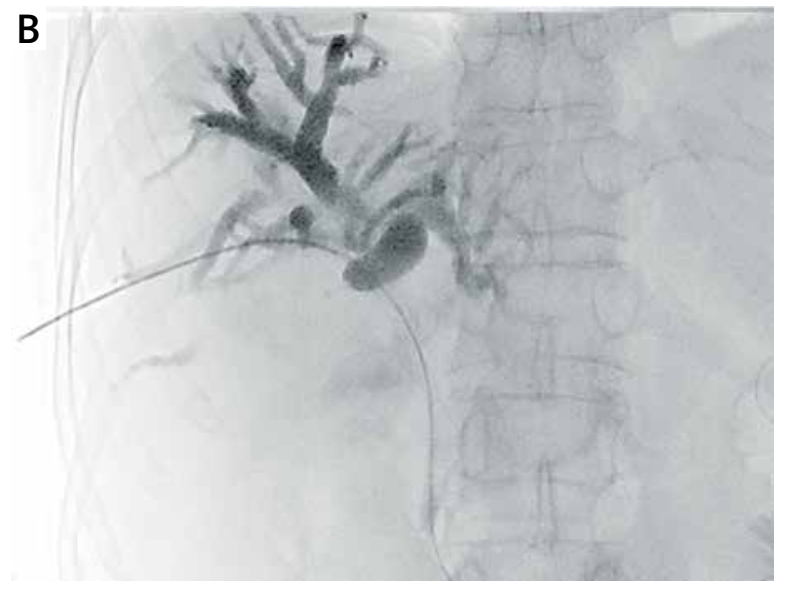

Photo 1. Stent treatment in a patient with BBS via PTCD. A - Cholangiography showing stricture region of the common bile duct. $\mathbf{B}$ - Guidewire advanced across stricture. $\mathbf{C}$ - The stricture resolved completely after a stent insertion

BDBSs is that they may degrade prematurely, failing to provide adequate support for expanding the narrowed biliary tract [16].

\section{Aim}

Up to now, several meta-analyses have been reported about different stents in the application of malignant biliary stricture. However, a systematic study comparing the efficacy of these stents in endoscopic treatment of BBS is still unavailable. Given the above descriptions, every stent has its pros and cons, and the selection is still controversial. Here, we performed a systematic review and meta-analysis to identify the roles of 3 common stents (MPSs, FCSEMSs, and BDBSs) in BBS management.

\section{Material and methods}

\section{Search strategy and study selection}

Two authors systematically searched the PubMed, Web of Science, Cochrane Library, and 
Wiley Library databases for studies published from 1 January 2010 to 12 August 2020, by using the following search terms: BBS, benign biliary stenosis, stents. A manual search through the reference of included studies was also carried out to identify the potentially relevant studies.

The studies enrolled in this meta-analysis were required to be randomized controlled trials (RCTs), case-controlled trials (CCTs), cohort studies, or case series studies, which met the following criteria: (1) studies including patients with BBS and treated with one of the types of stents (MPSs, FCSEMSs, or BDBSs) using endoscopic or percutaneous insertion; (2) studies carried out on humans; and (3) studies written in English. The exclusion criteria were: (1) case reports; (2) studies including minors; (3) studies including populations suffering from other complications, such as cystic duck leak or biliary leakage; and (4) unpublished data or data published in abstract form only.

\section{Data extraction}

Two authors independently extracted all relevant data from included studies, including publication year, study design, participant characteristics, stent types, implant methods, and follow-up duration. The primary outcome was the clinical success rate, defined as no record of unscheduled interventions, stricture relapse, or change in treatment strategy during the follow-up time. The secondary outcomes included technical success rate, treatment success rate, time to recurrence, adverse events, and intervention frequency. Technical success referred to stents that were successfully implanted at the final cholangiography and then removed successfully. Treatment success referred to BBS that was resolved at stent removal demonstrated by cholangiography or hepatic functional test.

\section{Quality assessment}

The criteria used to assess the quality of RCTs are described in Chapter 8 of the Cochrane Handbook for Systematic Review of Intervention [17]. The risk of bias from CCTs, cohort studies, and case series studies was assessed based on the Newcastle Ottwan Scale (NOS) [18]. Two reviewers independently performed the quality assessment, and all disagreements were resolved by discussion.

\section{Statistical analysis}

The pooled estimate and 95\% confidence interval $(\mathrm{Cl})$ were calculated by using a random effects model. The proportion was calculated for dichotomous variables, as well as the mean value for continuous outcomes. Heterogeneity among studies was assessed with the $R^{2}$ statistic and $\chi^{2}$ test. The result of the $R^{2}$ statistic ranged from $0 \%$ to $100 \%$, and we considered $l^{2}$ over $50 \%$ as a high degree of heterogeneity. Analysis was done by using Stata 16, in which $p<0.05$ indicated statistical significance.

\section{Ethics and dissemination}

Ethical approval and patient consent were not required because this was a systematic review and meta-analysis, all data in this study came from published literature and did not involve patients.

\section{Results}

\section{Literature search}

According to the aforementioned inclusion and exclusion criteria, we identified a total of 1148 potentially relevant articles in the present meta-analysis. After removal of duplicates, 880 studies remained. After screening the titles and abstracts, 832 irrelevant studies were excluded. After evaluating the full text of the remaining studies, 28 studies were eligible for this meta-analysis. We added 1 relevant study after review of the reference list, and a total of 29 studies ( 1 RCT, 1 CCT, and 27 case series studies) were included. We divided the RCT and CCT (each containing 2 kinds of stents) into 2 separate studies individually, and the final number of included studies was 31 . The selection process was recorded to complete a PRISMA flow diagram (Figure 1).

\section{Study characteristics}

The 31 included studies, with 1604 patients, were published from 1 January 2010 to 12 August 2020. These participants were divided into 3 groups according to the stent type: 7 studies with 363 patients used BDBSs, 19 studies with 965 patients used FCSEMSs, and 5 studies with 276 patients used MPSs. The main characteristics of included studies are shown in Table I [19-36]. Of these studies, 20 were prospective studies, and the others were retrospective studies. The stents were implanted by endoscopic retrograde cholangiopancreatography 


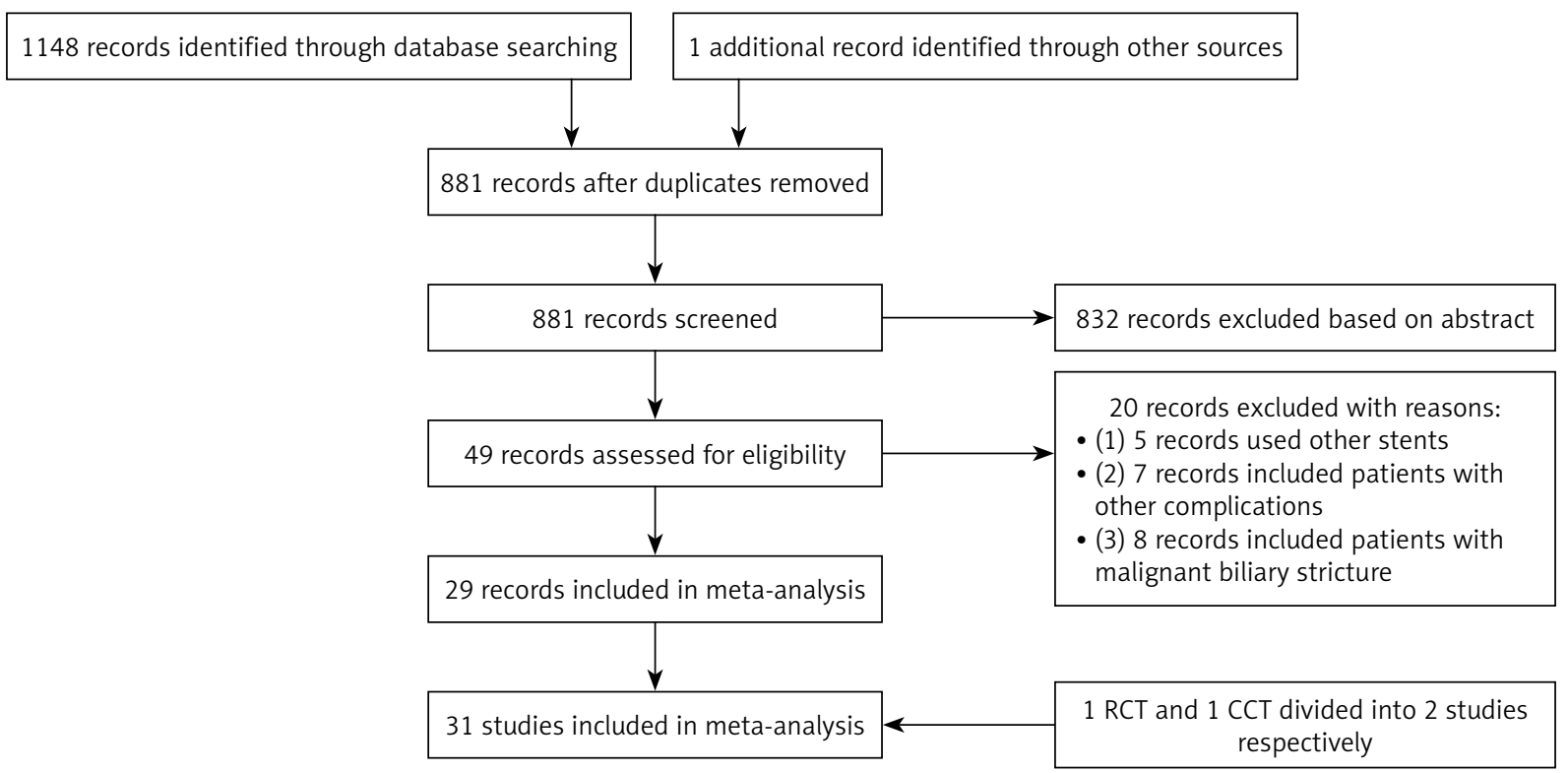

Figure 1. Study flow diagram

(ERCP) in 25 studies and by percutaneous transhepatic cholangiography (PTC) in the others.

\section{Quality assessment}

We assessed the qualities of included studies using the NOS. The items derived from this scale included 5 questions, representativeness of samples, accuracy of diagnosis, duration of follow-up, integrity of reported date, and ascertainment of outcome. The study was awarded 1 point for meeting each question, and scores of $<3,4$, and 5 corresponded to low, moderate, and high quality $[37,38]$. The quality assessment results of 31 included studies are shown in Table II. Sixteen studies were high, 13 studies were moderate, and 2 were low quality.

\section{Clinical success}

Thirty included studies reported the clinical success rate. From the result of this meta-analysis, as shown in Figure 2, the clinical success was most likely to be achieved when using BDBSs $(0.76,95 \% \mathrm{Cl}$ : $0.71-0.80)$. The pooled clinical success rate of MPSs was 0.69 (95\% Cl: 0.63-0.74), which was higher than that of FCSEMSs $(0.66,95 \% \mathrm{Cl}: 0.60-0.72)$. The heterogeneity was evaluated as low for BDBSs $\left(I^{2}=\right.$ $0.00 \%$ and $p=0.74)$, high for FCSEMSs $\left(R^{2}=65.82 \%\right.$ and $p<0.001)$, and low for MPSS $\left(I^{2}=0.00 \%\right.$ and $p=0.62)$. The high heterogeneity of the FCSEMS group might relate to the inclusion of 3 studies (Po- ley 2020, Moon 2012, and Lakhtakia 2019). In Poley 2020 , the stents were scheduled to be removed at 4-6 months (median: 153 days) after implantation, obviously shorter than other studies. In Lakhtakia 2019, 62 patients were lost to follow-up, accounting for a large proportion of included samples (52.6\%). In Moon 2012, the used stents had a convex margin at both ends, somewhat different from common FCSEMSs. After exclusion of these 3 studies, the lever of heterogeneity became low $\left(R^{2}=22.24 \%\right.$ and $p=0.21$ ), but the pooled clinical success rate of FCSEMSs was little changed $(0.67,95 \% \mathrm{Cl}$ : $0.63-$ 0.71) (Figure 3).

\section{Adverse events}

All 31 included studies reported the adverse events caused by the inserted stents, and the detailed information is summarized in Tables III and IV. From the result of this meta-analysis, shown in Figure 4 , the BDBS group showed the lowest overall incidence of adverse events (0.31, 95\% Cl: 0.12-0.54), followed by the FCSEMS group $(0.40,95 \% \mathrm{Cl}$ : $0.31-$ $0.50)$, and MPS group $(0.46,95 \% \mathrm{Cl}: 0.31-0.61)$. Due to the wide variation of categories of reported adverse events among included studies, the heterogeneities in all 3 groups were high, as expected $\left(R^{2}=\right.$ $92.71 \%$ and $p<0.001$ for BDBSs, $R^{2}=88.28 \%$ and $p<0.001$ for FCSEMSs, $p=76.54 \%$ and $p<0.001$ for MPSs). Therefore, we further performed a subgroup analysis according to 4 common adverse 


\begin{tabular}{|c|c|c|c|c|c|c|c|c|c|c|c|c|c|c|c|c|c|}
\hline 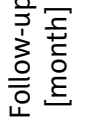 & 芦 & $\stackrel{*}{\stackrel{*}{\sim}}$ & $\stackrel{*}{\sim}$ & $\stackrel{*}{\sim}$ & 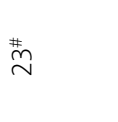 & $\stackrel{\text { \# }}{\stackrel{\text { N }}{\sim}}$ & 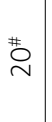 & $\begin{array}{l}\underbrace{*}_{0} \\
\stackrel{\leftrightarrow}{\oplus}\end{array}$ & $\stackrel{\substack{* \\
i \\
i n}}{n}$ & \begin{tabular}{l|l}
$*$ \\
0 \\
0
\end{tabular} & \begin{tabular}{l|l}
$*$ \\
$\stackrel{\infty}{\infty}$
\end{tabular} & $\stackrel{*}{*}$ & $\stackrel{*}{n}$ & $\sum$ & $\stackrel{*}{\sharp}$ & $\stackrel{*}{\exists}$ & * \\
\hline 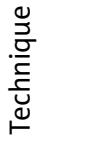 & $\underset{\swarrow}{\swarrow}$ & $\breve{\hbar}$ & $\begin{array}{l}\text { Ù } \\
\stackrel{x}{\Psi}\end{array}$ & 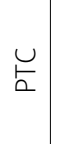 & $\breve{\llcorner}$ & $\breve{\curvearrowleft}$ & 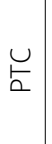 & 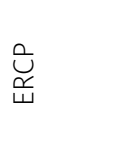 & $\begin{array}{l}\stackrel{\vartheta}{\breve{s}} \\
\stackrel{\sim}{\Psi}\end{array}$ & $\begin{array}{l}\stackrel{O}{u} \\
\stackrel{\sim}{\Psi}\end{array}$ & 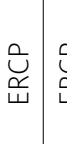 & $\underset{\stackrel{u}{\Psi}}{\stackrel{u}{\Psi}}$ & 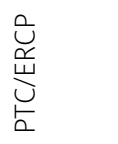 & 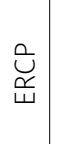 & $\begin{array}{l}\stackrel{u}{u} \\
\stackrel{u}{u}\end{array}$ & 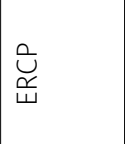 & 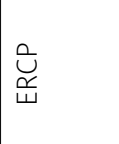 \\
\hline 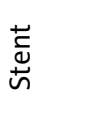 & $\begin{array}{l}\widetilde{\sim} \\
\stackrel{\tilde{\omega}}{\infty}\end{array}$ & $\begin{array}{l}\widetilde{\nu} \\
\stackrel{\rho}{\infty}\end{array}$ & $\begin{array}{l}\widetilde{\omega} \\
\stackrel{\tilde{\nu}}{\infty}\end{array}$ & $\begin{array}{l}\tilde{\tilde{~}} \\
\tilde{\omega}\end{array}$ & $\begin{array}{l}\widetilde{\tilde{~}} \\
\tilde{\infty}\end{array}$ & $\begin{array}{l}\widetilde{u} \\
\tilde{\omega} \\
\tilde{\infty}\end{array}$ & $\begin{array}{l}\sim \\
\tilde{n} \\
0 \\
0\end{array}$ & 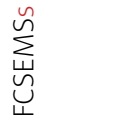 & 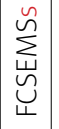 & 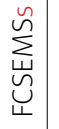 & 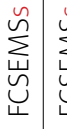 & $\sum_{\breve{u}}^{\breve{u n}}$ & 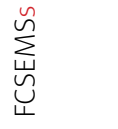 & 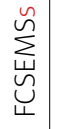 & $\sum_{\breve{u}}^{\breve{u n}}$ & 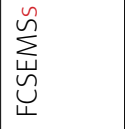 & 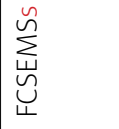 \\
\hline 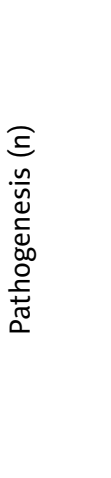 & 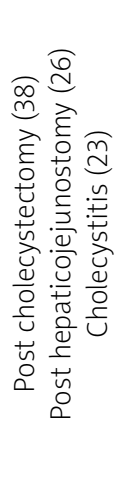 & 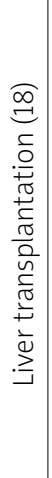 & 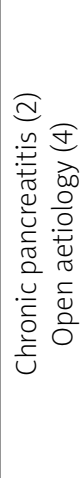 & 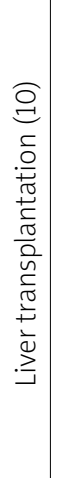 & 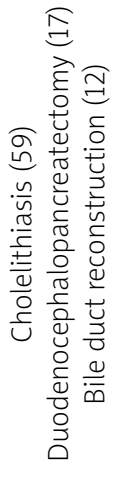 & 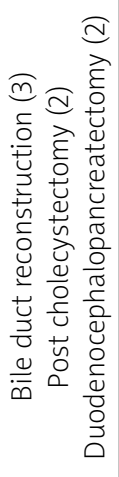 & 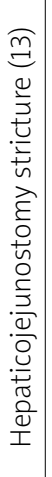 & 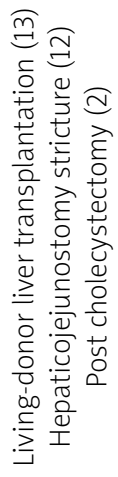 & 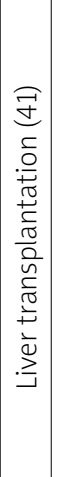 & 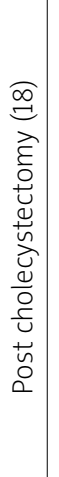 & 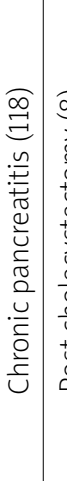 & 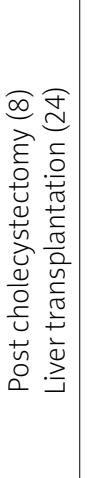 & 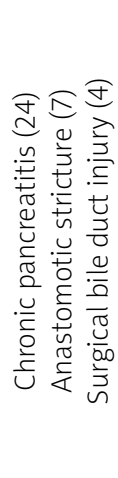 & 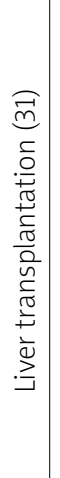 & 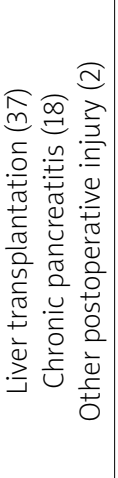 & 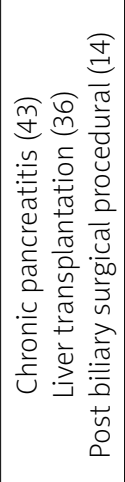 & 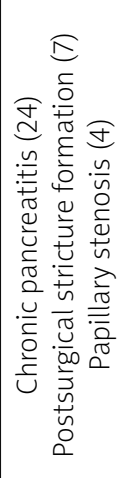 \\
\hline 离 & $\begin{array}{l}\circ \\
\stackrel{2}{f} \\
\dot{f}\end{array}$ & $\stackrel{m}{\stackrel{m}{n}}$ & $\stackrel{\sim}{f}$ & $\stackrel{\curvearrowright}{\Rightarrow}$ & $\begin{array}{l}\overrightarrow{0} \\
\vec{\sigma} \\
+\end{array}$ & $\stackrel{\substack{m \\
\sim}}{\sim}$ & $\frac{\Delta}{\sigma}$ & $\stackrel{\ominus}{\Xi}$ & $\stackrel{i}{N}$ & $\underset{\nearrow}{\stackrel{\Xi}{\nearrow}}$ & 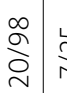 & $\stackrel{\varrho}{\aleph}$ & $\stackrel{m}{\stackrel{m}{O}}$ & 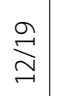 & 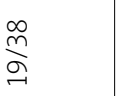 & $\stackrel{\infty}{\stackrel{\infty}{\sim}}$ & $\underset{\underset{J}{J}}{\stackrel{J}{J}}$ \\
\hline 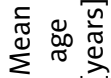 & 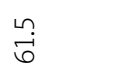 & $\begin{array}{l}m \\
\infty \\
\infty\end{array}$ & 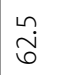 & 8 & in & $\begin{array}{l}\text { 禹 } \\
\stackrel{\leftrightarrow}{n}\end{array}$ & $\begin{array}{c}\widehat{\infty} \\
\stackrel{m}{m}\end{array}$ & $\hat{0}$ & $\begin{array}{l}\hat{0} \\
\stackrel{\omega}{n}\end{array}$ & 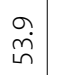 & $\vec{i}$ & กn & $\underset{\substack{\infty \\
\infty}}{\stackrel{\infty}{n}}$ & $\stackrel{\bullet}{\circ}$ & 管 & $\stackrel{+}{\stackrel{*}{*}}$ & in \\
\hline 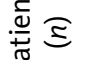 & ભે & $\stackrel{\infty}{\sim}$ & 0 & 의 & $\stackrel{0}{a}$ & in & $\eta$ & $\stackrel{\text { p }}{ }$ & $\vec{F}$ & $\stackrel{\infty}{\sim}$ & $\stackrel{\infty}{\Rightarrow}:$ & $\tilde{m}$ & q & $\vec{m}$ & in & $\tilde{\sigma}$ & $\stackrel{\infty}{m}$ \\
\hline 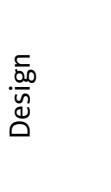 & 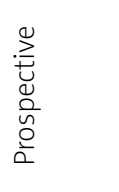 & 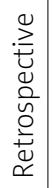 & 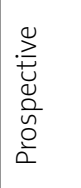 & 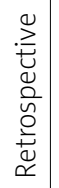 & 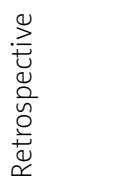 & 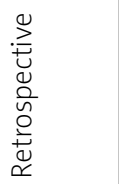 & 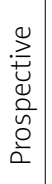 & 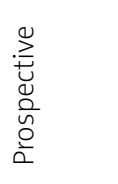 & 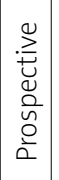 & 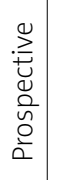 & 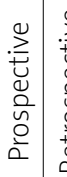 & 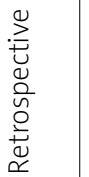 & 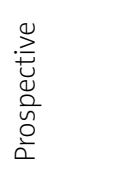 & 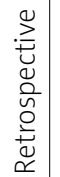 & 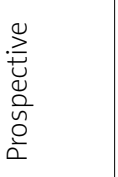 & 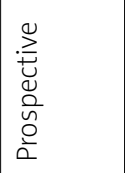 & 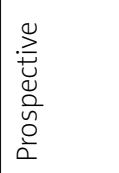 \\
\hline $\begin{array}{l}\text { 离 } \\
\text { in }\end{array}$ & 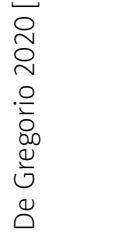 & 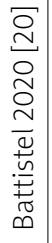 & 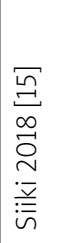 & $\begin{array}{l}\bar{n} \\
\infty \\
0 \\
0 \\
0 \\
\tilde{n} \\
0 \\
0\end{array}$ & 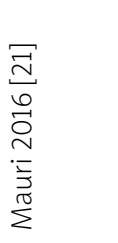 & 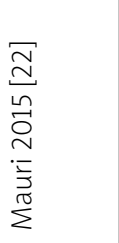 & 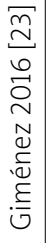 & 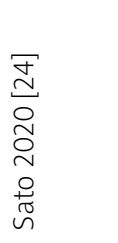 & 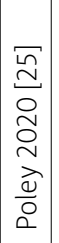 & 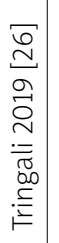 & 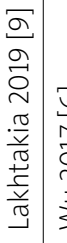 & 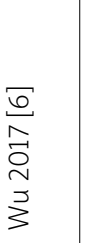 & 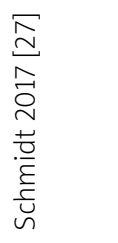 & 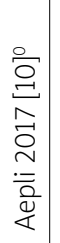 & 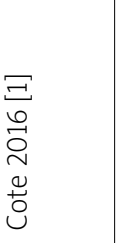 & 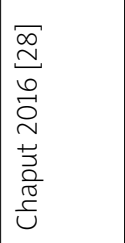 & 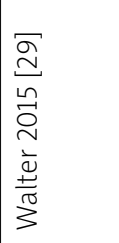 \\
\hline
\end{tabular}




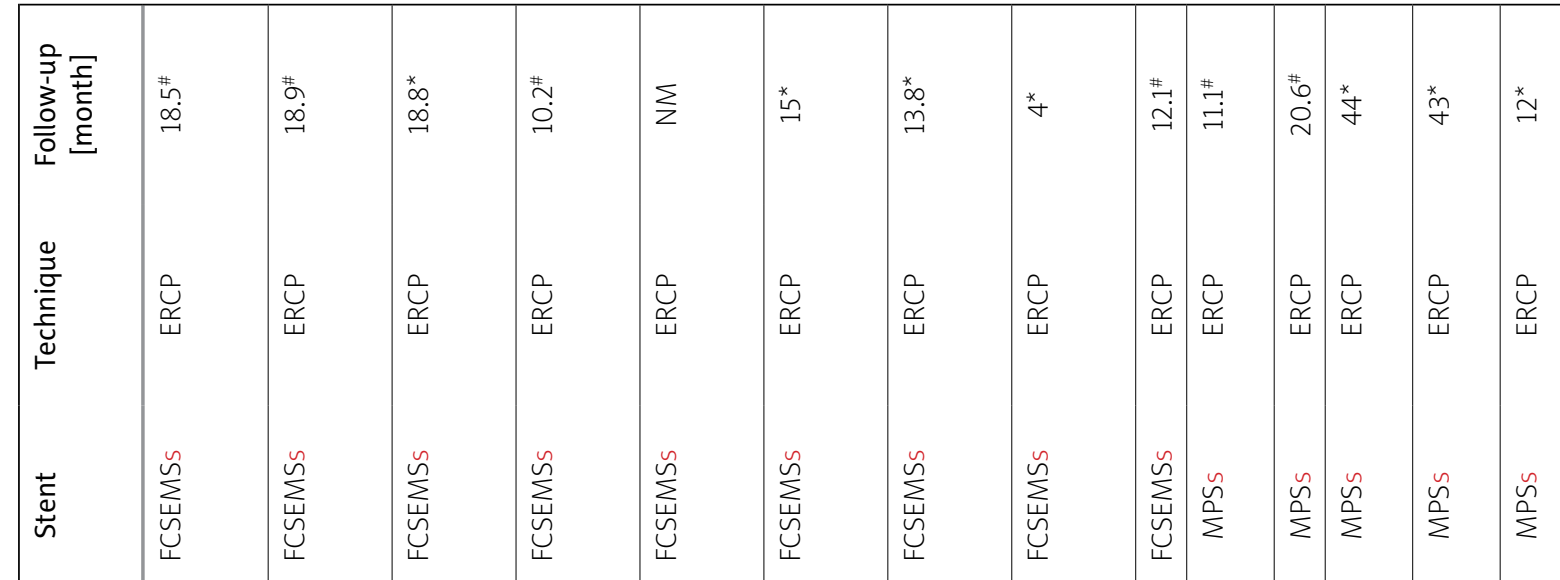

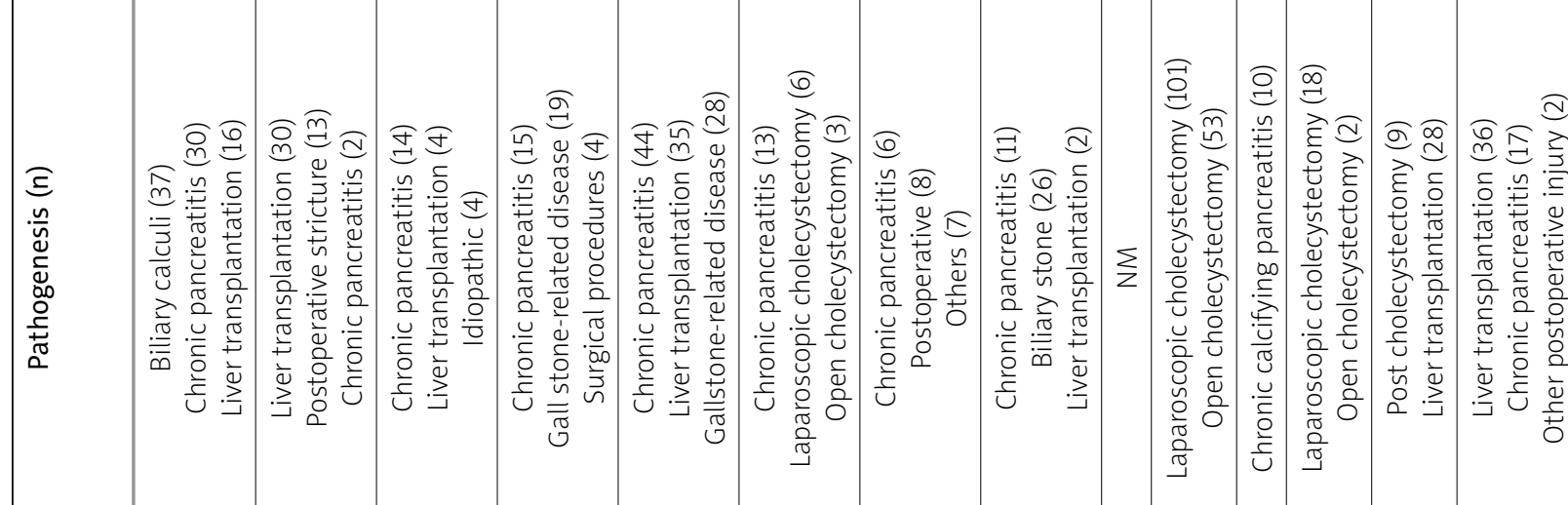

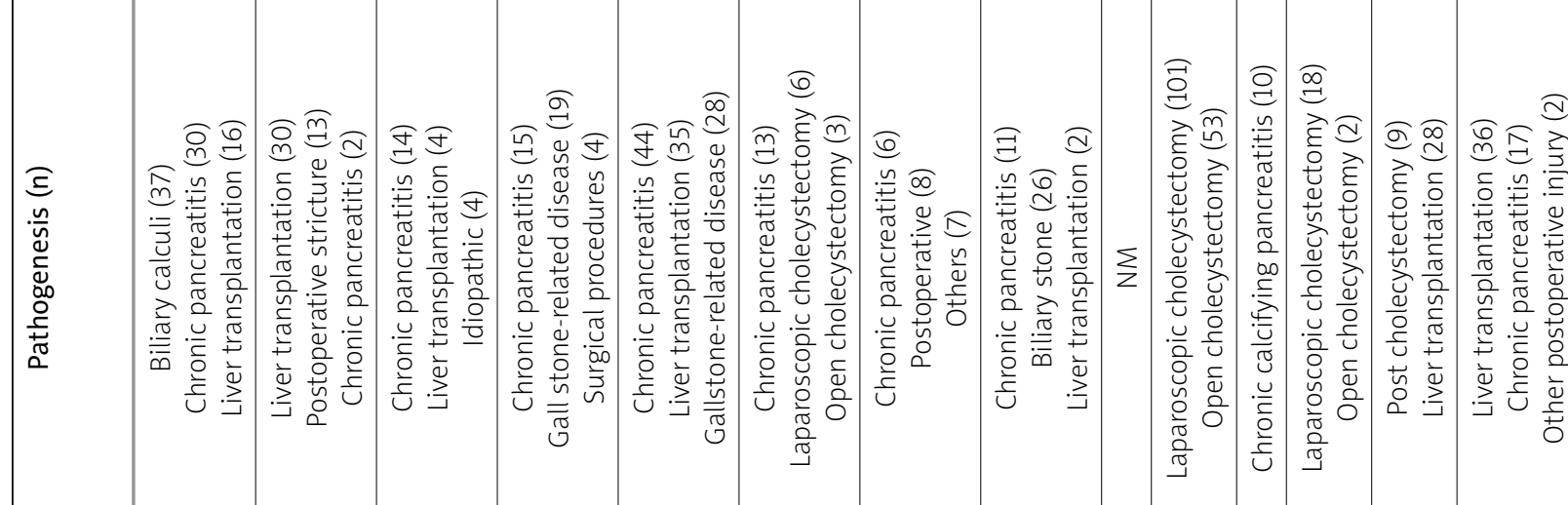

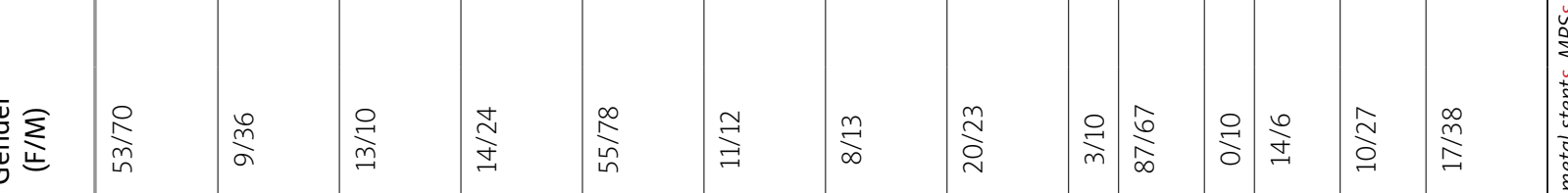

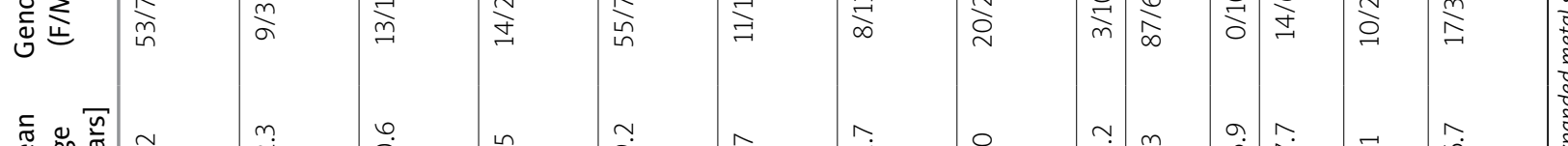

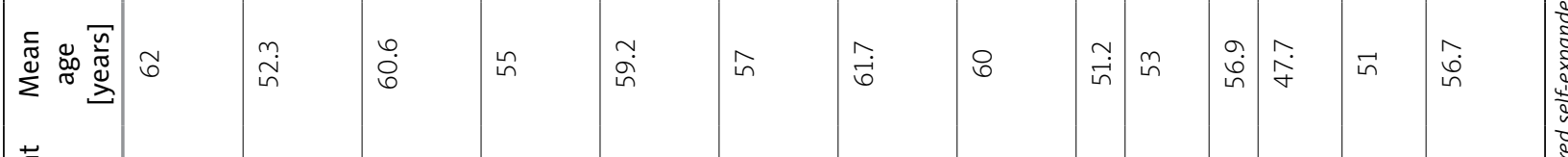

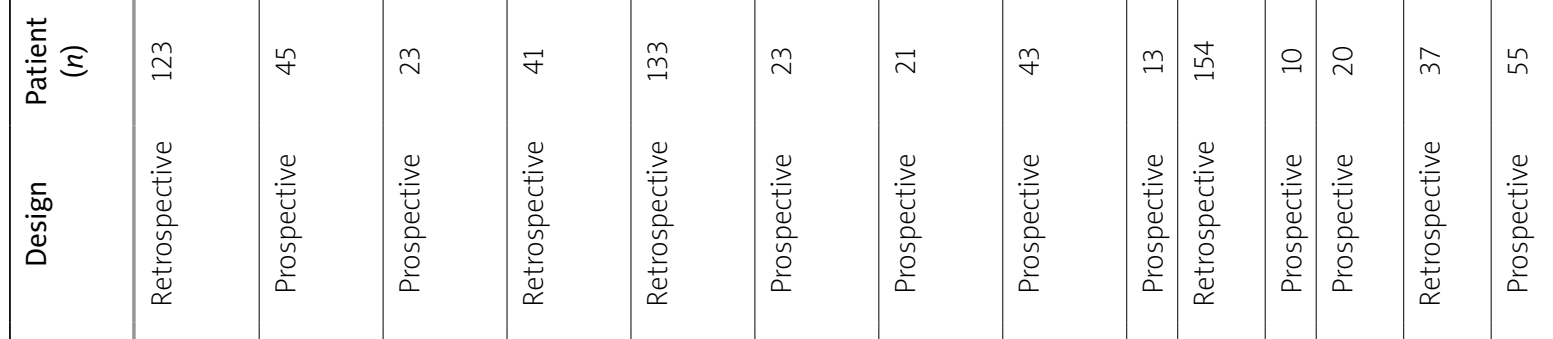

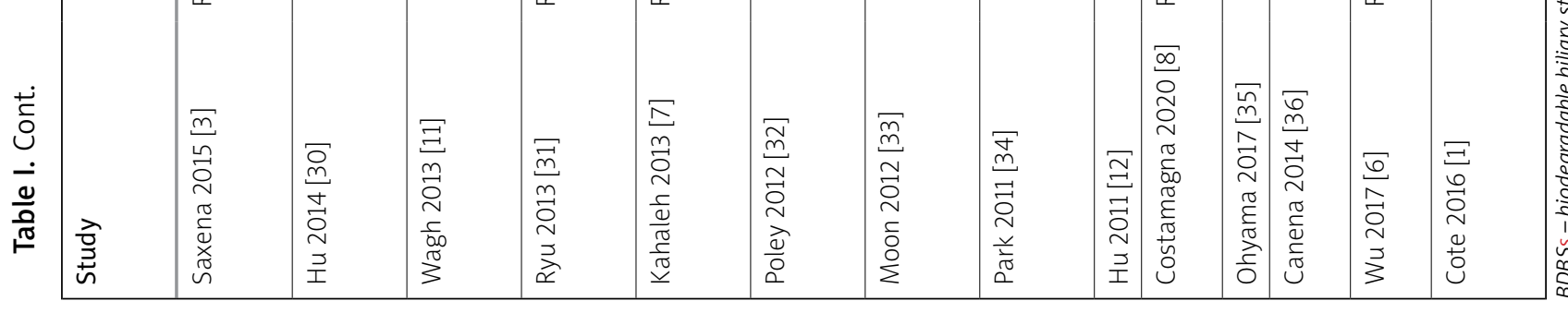


Table II. Results of quality assessment

\begin{tabular}{|c|c|c|c|c|c|c|}
\hline Study & Question 1 & Question 2 & Question 3 & Question 4 & Question 5 & Quality \\
\hline Battistel 2020 [20] & Yes & Yes & Yes & Yes & Yes & High \\
\hline De Gregorio 2020 [19] & Yes & Yes & Yes & Yes & Yes & High \\
\hline Siiki 2018 [15] & Yes & Yes & Yes & Yes & Yes & High \\
\hline Dopazo 2018 [5] & Yes & Yes & No & Yes & Yes & Moderate \\
\hline Mauri 2016 [21] & Yes & Yes & Yes & Yes & Yes & High \\
\hline Mauri 2015 [22] & Yes & Yes & No & Yes & Yes & Moderate \\
\hline Giménez 2016 [23] & Yes & Yes & Yes & Yes & Yes & High \\
\hline Sato 2020 [24] & Yes & Yes & Yes & Yes & Yes & High \\
\hline Poley 2020 [25] & Yes & Yes & Yes & Yes & Yes & High \\
\hline Tringali 2019 [26] & Yes & yes & No & Yes & Yes & Moderate \\
\hline Lakhtakia 2019 [9] & Yes & Yes & Yes & Yes & Yes & High \\
\hline Wu 2017 [6] & Yes & Yes & Yes & Yes & Yes & High \\
\hline Schmidt 2017 [27] & Yes & Yes & No & Yes & Yes & Moderate \\
\hline Aepli 2107 [10] & Yes & Yes & No & No & Yes & Low \\
\hline Cote 2016 [1] & Yes & Yes & Yes & Yes & Yes & High \\
\hline Chaput 2016 [28] & Yes & Yes & No & Yes & Yes & Moderate \\
\hline Walter 2015 [29] & Yes & Yes & No & Yes & Yes & Moderate \\
\hline Saxena 2015 [3] & Yes & Yes & Yes & Yes & Yes & High \\
\hline Hu 2014 [30] & Yes & Yes & No & Yes & Yes & Moderate \\
\hline Wagh 2013 [11] & Yes & Yes & Yes & Yes & Yes & High \\
\hline Ryu 2013 [31] & Yes & Yes & No & Yes & Yes & Moderate \\
\hline Kahaleh 2013 [7] & Yes & Yes & No & No & Yes & Low \\
\hline Poley 2012 [32] & Yes & Yes & Yes & Yes & Yes & High \\
\hline Moon 2012 [33] & Yes & Yes & No & Yes & Yes & Moderate \\
\hline Park 2011 [34] & Yes & Yes & No & Yes & Yes & Moderate \\
\hline Hu 2011 [12] & Yes & Yes & No & Yes & Yes & Moderate \\
\hline Costamagna 2020 [8] & Yes & Yes & No & Yes & Yes & Moderate \\
\hline Ohyama 2017 [35] & Yes & Yes & Yes & Yes & Yes & High \\
\hline Wu 2017 [6] & Yes & Yes & Yes & Yes & Yes & High \\
\hline Cote 2016 [1] & Yes & Yes & Yes & Yes & Yes & High \\
\hline Canena 2014 [36] & Yes & Yes & No & Yes & Yes & Moderate \\
\hline
\end{tabular}

Question 1: Did the patients represent all of the cases of the medical centre? Question 2: Was the diagnosis correctly made? Question 3: Was the follow-up long enough for outcomes to occur? Question 4: Were all important data cited in the report? Question 5: Was the outcome correctly reported?

events, including abdominal pain, cholangitis, pancreatitis, and stent migration.

\section{Abdominal pain}

For abdominal pain, all 31 included studies reported this complication. After the meta-analysis in Figure 5, the lowest rate was found in the BDBS group (0.01, 95\% Cl: 0.00-0.07), although the differences among the groups were not significant. The pooled rate in the FCSEMS group was $0.03(95 \% \mathrm{Cl}$ : $0.01-0.06)$, similar to that in the MPS group $(0.03$, 95\% Cl: 0.00-0.12). The heterogeneity result was high for all 3 groups $\left(R^{2}=69.39 \%\right.$ and $p<0.001$ for BDBSs, $l^{2}=78.47 \%$ and $p<0.001$ for FCSEMSs, $l^{2}=$ $78.44 \%$ and $p<0.001$ for MPSs). 


\section{BDBSS}

Battistel 2020

De Gregorio 2020

Siiki 2018

Dopazo 2018

Mauri 2016

Mauri 2015

Giménez 2016

Subtotal $\left(I^{2}=0.00 \%, p=0.74\right)$

\section{FCSEMSS}

Sato 2020

Poley 2020

Tringali 2019

Lakhtakia 2019

Wu 2017

Schmidt 2017

Aepli 2017

Cote 2016

Chaput 2016

Walter 2015

Saxena 2015

Hu 2014

Wagh 2013

Ryu 2013

Poley 2012

Moon 2012

Park 2011

Hu 2011

Subtotal $\left(I^{2}=65.82 \%, p<0.001\right)$

\section{MPSs}

Costamagna 2020

Ohyama 2017

Canena 2014

Wu 2017

Cote 2016

Subtotal $\left(I^{2}=0.00 \%, p=0.62\right)$

Heterogeneity between groups: $p=0.049$

Overall $\left(I^{2}=57.82 \%, p<0.001\right)$

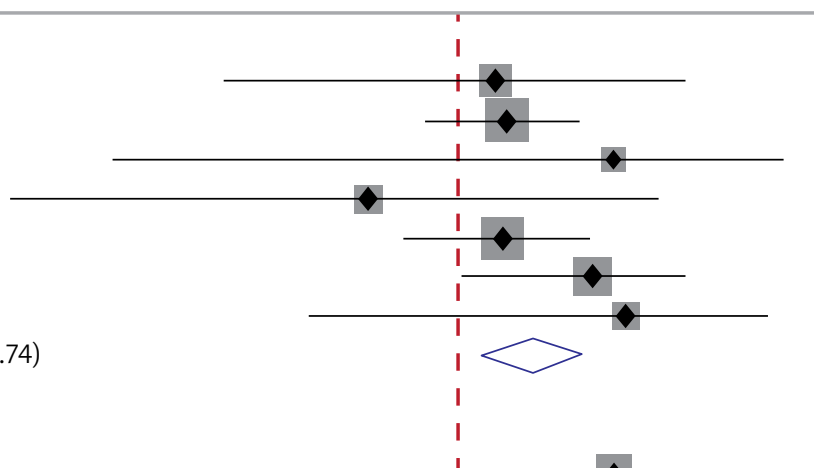

$0.72(0.47,0.90) \quad 2.28$

$0.73(0.66,0.80) \quad 5.36$

$0.83(0.36,1.00) \quad 1.03$

$0.60(0.26,0.88) \quad 1.52$

$0.73(0.63,0.81) \quad 4.98$

$0.81(0.69,0.90) \quad 4.15$

$0.85(0.55,0.98) \quad 1.83$

$0.76(0.71,0.80) \quad 21.15$

$0.83(0.65,0.94) \quad 3.07$

$0.49(0.33,0.65) \quad 3.58$

$0.61(0.36,0.83) \quad 2.28$

$0.47(0.38,0.57) \quad 5.10$

$0.75(0.57,0.89) \quad 3.17$

$0.53(0.38,0.69) \quad 3.65$

$0.71(0.52,0.86) \quad 3.12$

$0.61(0.48,0.74) \quad 4.10$

$0.62(0.51,0.72) \quad 4.79$

$0.58(0.41,0.74) \quad 3.45$

$0.72(0.63,0.79) \quad 5.15$

$0.62(0.47,0.76) \quad 3.73$

$0.65(0.43,0.84) \quad 2.65$

$0.78(0.62,0.89) \quad 3.58$

$0.61(0.39,0.80) \quad 2.65$

$0.95(0.76,1.00) \quad 2.51$

$0.65(0.49,0.79) \quad 3.65$

$0.85(0.55,0.98) \quad 1.83$

$0.66(0.60,0.72) \quad 62.05$

$0.69(0.61,0.76) \quad 5.39$

$0.60(0.26,0.88) \quad 1.52$

$0.80(0.56,0.94) \quad 2.43$

$0.70(0.53,0.84) \quad 3.41$

$0.62(0.48,0.75) \quad 4.05$

$0.69(0.63,0.74) \quad 16.79$

$0.69(0.64,0.73) \quad 100.00$

\begin{tabular}{cc|c|c|c}
\hline & & & \\
0.25 & 0.50 & 0.75 & 1 & 1.00
\end{tabular}

Figure 2. Forest plot comparing clinical success rate in different groups using effect size (ES) with ordinary weighting

ES - effect size, $\mathrm{Cl}$ - confidence interval.

\section{Cholangitis}

For cholangitis, all 31 included studies reported this complication. After the meta-analysis in Figure 6 , the lowest rate was found in the BDBS group
(0.05, 95\% Cl: $0.00-0.20)$. The pooled rate in the FCSEMS group was 0.06 (95\% Cl: 0.03-0.10), lower than that in the MPS group $(0.11,95 \% \mathrm{Cl}: 0.02-$ 0.23). The heterogeneity was high for all 3 groups 


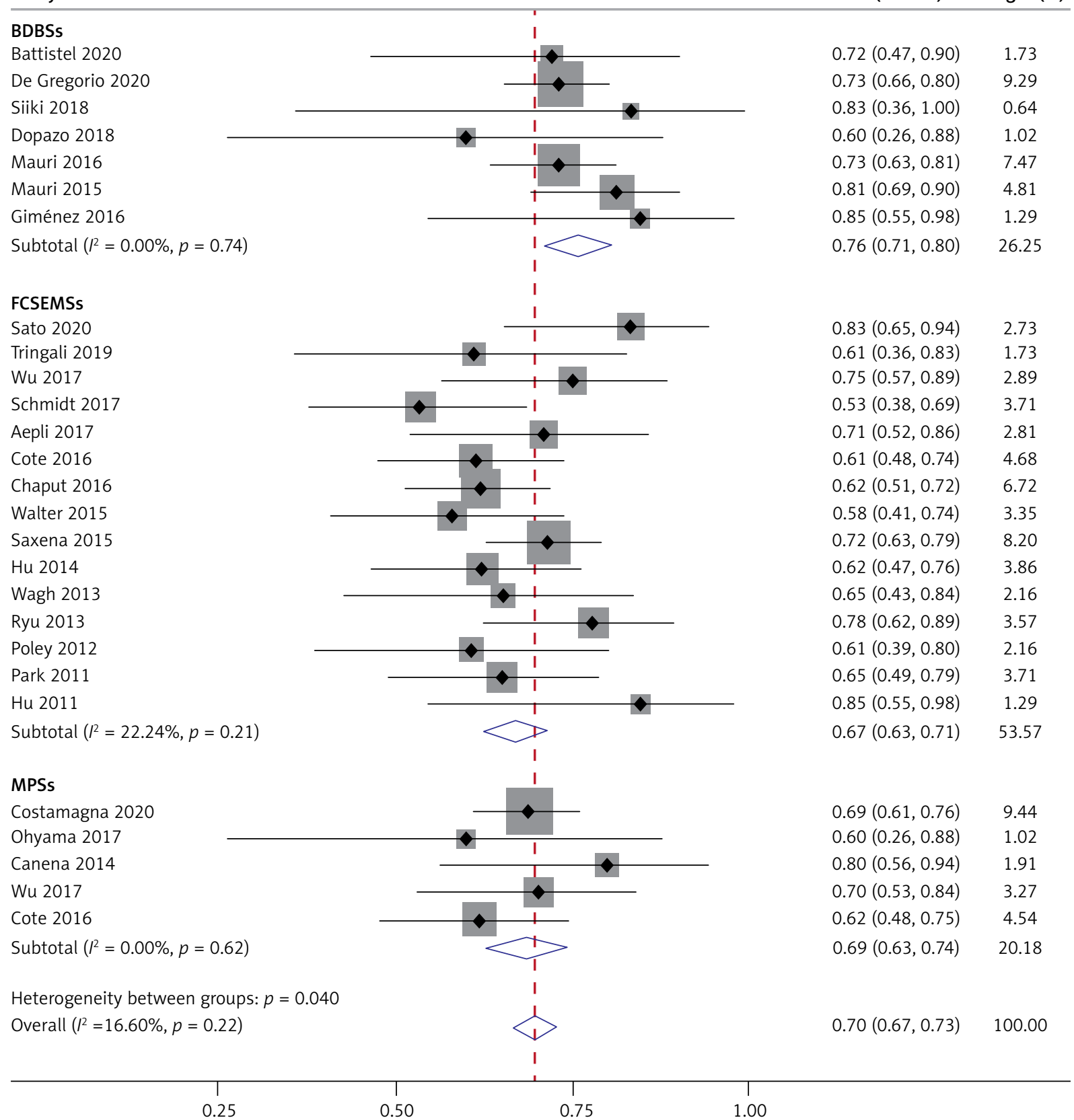

Figure 3. Forest plot comparing clinical success rate in different groups after excluding three studies (Poley 2020, Moon 2012, and Lakhtakia 2019)

ES - effect size, $\mathrm{Cl}$ - confidence interval.

$\left(I^{2}=92.01 \%\right.$ and $p<0.001$ for BDBSs, $I^{2}=77.63 \%$ and $p<0.001$ for FCSEMSs, and $R^{2}=79.74 \%$ and $p<0.001$ for MPSs).

\section{Stent migration}

All 31 studies reported stent migration. After the meta-analysis in Figure 7, the lowest rate was found in the BDBS group $(0.01,95 \% \mathrm{Cl}: 0.00-0.02)$, although the differences among the groups were not significant. The pooled rate in the MPS group was 0.07 (95\% Cl: 0.00-0.20), which was lower than that in the FCSEMS group $(0.12,95 \% \mathrm{Cl}: 0.07-0.19)$. The heterogeneity was low for the BDBS group $\left(I^{2}=0.0 \%\right.$ and $p=0.99)$ and high for the other 2 groups $\left(I^{2}=\right.$ 
Table III. Summary of common adverse events

\begin{tabular}{|c|c|c|c|c|c|c|}
\hline Stent & Adverse event & Study & Patient $(n)$ & Event $(n)$ & Incidence & Pooled date \\
\hline \multirow[t]{28}{*}{ BDBSS } & \multirow[t]{7}{*}{ Abdominal pain } & Battistel 2020 [20] & 18 & 0 & $0.00 \%$ & \multirow[t]{7}{*}{$3.03 \%$} \\
\hline & & De Gregorio 2020 [19] & 150 & 7 & $4.67 \%$ & \\
\hline & & Siiki 2018 [15] & 6 & 2 & $33.33 \%$ & \\
\hline & & Dopazo 2018 [5] & 10 & 1 & $10.00 \%$ & \\
\hline & & Mauri 2016 [21] & 107 & 0 & $0.00 \%$ & \\
\hline & & Mauri 2015 [22] & 59 & 0 & $0.00 \%$ & \\
\hline & & Giménez 2016 [23] & 13 & 1 & $7.69 \%$ & \\
\hline & \multirow[t]{7}{*}{ Cholangitis } & Battistel 2020 [20] & 18 & 0 & $0.00 \%$ & \multirow[t]{7}{*}{$8.54 \%$} \\
\hline & & De Gregorio 2020 [19] & 150 & 0 & $0.00 \%$ & \\
\hline & & Siiki 2018 [15] & 6 & 3 & $50.00 \%$ & \\
\hline & & Dopazo 2018 [5] & 10 & 0 & $0.00 \%$ & \\
\hline & & Mauri 2016 [21] & 107 & 26 & $24.30 \%$ & \\
\hline & & Mauri 2015 [22] & 59 & 0 & $0.00 \%$ & \\
\hline & & Giménez 2016 [23] & 13 & 2 & $15.38 \%$ & \\
\hline & \multirow[t]{7}{*}{ Pancreatitis } & Battistel 2020 [20] & 18 & 0 & $0.00 \%$ & \multirow[t]{7}{*}{$0.83 \%$} \\
\hline & & De Gregorio 2020 [19] & 150 & 0 & $0.00 \%$ & \\
\hline & & Siiki 2018 [15] & 6 & 1 & $16.67 \%$ & \\
\hline & & Dopazo 2018 [5] & 10 & 1 & $10.00 \%$ & \\
\hline & & Mauri 2016 [21] & 107 & 1 & $0.93 \%$ & \\
\hline & & Mauri 2015 [22] & 59 & 0 & $0.00 \%$ & \\
\hline & & Giménez 2016 [23] & 13 & 0 & $0.00 \%$ & \\
\hline & \multirow[t]{7}{*}{ Stent migration } & Battistel 2020 [20] & 18 & 0 & $0.00 \%$ & \multirow[t]{7}{*}{$2.20 \%$} \\
\hline & & De Gregorio 2020 [19] & 150 & 5 & $3.33 \%$ & \\
\hline & & Siiki 2018 [15] & 6 & 0 & $0.00 \%$ & \\
\hline & & Dopazo 2018 [5] & 10 & 0 & $0.00 \%$ & \\
\hline & & Mauri 2016 [21] & 107 & 2 & $1.87 \%$ & \\
\hline & & Mauri 2015 [22] & 59 & 1 & $1.69 \%$ & \\
\hline & & Giménez 2016 [23] & 13 & 0 & $0.00 \%$ & \\
\hline \multirow[t]{13}{*}{ FCSEMSS } & \multirow[t]{13}{*}{ Abdominal pain } & Sato 2020 [24] & 30 & 0 & $0.00 \%$ & \multirow[t]{13}{*}{$5.28 \%$} \\
\hline & & Poley 2020 [25] & 41 & 4 & $9.76 \%$ & \\
\hline & & Tringali 2019 [26] & 18 & 0 & $0.00 \%$ & \\
\hline & & Lakhtakia 2019 [9] & 118 & 9 & $7.63 \%$ & \\
\hline & & Wu 2017 [6] & 32 & 0 & $0.00 \%$ & \\
\hline & & Schmidt 2017 [27] & 43 & 1 & $2.33 \%$ & \\
\hline & & Aepli 2017 [10] & 31 & 0 & $0.00 \%$ & \\
\hline & & Cote 2016 [1] & 57 & 8 & $14.04 \%$ & \\
\hline & & Chaput 2016 [28] & 92 & 4 & $4.35 \%$ & \\
\hline & & Walter 2015 [29] & 38 & 0 & $0.00 \%$ & \\
\hline & & Saxena 2015 [3] & 123 & 0 & $0.00 \%$ & \\
\hline & & Hu 2014 [30] & 45 & 0 & $0.00 \%$ & \\
\hline & & Wagh 2013 [11] & 23 & 1 & $4.35 \%$ & \\
\hline
\end{tabular}


Table III. Cont.

\begin{tabular}{|c|c|c|c|c|c|c|}
\hline Stent & Adverse event & Study & Patient $(n)$ & Event $(n)$ & Incidence & Pooled date \\
\hline \multirow[t]{42}{*}{ FCSEMSS } & \multirow[t]{6}{*}{ Abdominal pain } & Ryu 2013 [31] & 41 & 3 & $7.32 \%$ & \multirow[t]{6}{*}{$5.28 \%$} \\
\hline & & Kahaleh 2013 [7] & 133 & 8 & $6.02 \%$ & \\
\hline & & Poley 2012 [32] & 23 & 13 & $56.52 \%$ & \\
\hline & & Moon 2012 [33] & 21 & 0 & $0.00 \%$ & \\
\hline & & Park 2011 [34] & 43 & 0 & $0.00 \%$ & \\
\hline & & Hu 2011 [12] & 13 & 0 & $0.00 \%$ & \\
\hline & \multirow[t]{19}{*}{ Cholangitis } & Sato 2020 [24] & 30 & 5 & $16.67 \%$ & \multirow[t]{19}{*}{$7.25 \%$} \\
\hline & & Poley 2020 [25] & 41 & 10 & $24.39 \%$ & \\
\hline & & Tringali 2019 [2] & 18 & 6 & $33.33 \%$ & \\
\hline & & Lakhtakia 2019 [9] & 118 & 18 & $15.25 \%$ & \\
\hline & & Wu 2017 [6] & 32 & 4 & $12.50 \%$ & \\
\hline & & Schmidt 2017 [27] & 43 & 1 & $2.33 \%$ & \\
\hline & & Aepli 2017 [10] & 31 & 2 & $6.45 \%$ & \\
\hline & & Cote 2016 [1] & 57 & 2 & $3.51 \%$ & \\
\hline & & Chaput 2016 [28] & 92 & 6 & $6.52 \%$ & \\
\hline & & Walter 2015 [29] & 38 & 5 & $13.16 \%$ & \\
\hline & & Saxena 2015 [3] & 123 & 5 & $4.07 \%$ & \\
\hline & & Hu 2014 [30] & 45 & 1 & $2.22 \%$ & \\
\hline & & Wagh 2013 [11] & 23 & 0 & $0.00 \%$ & \\
\hline & & Ryu 2013 [31] & 41 & 0 & $0.00 \%$ & \\
\hline & & Kahaleh 2013 [7] & 133 & 0 & $0.00 \%$ & \\
\hline & & Poley 2012 [32] & 23 & 3 & $13.04 \%$ & \\
\hline & & Moon 2012 [33] & 21 & 0 & $0.00 \%$ & \\
\hline & & Park 2011 [34] & 43 & 2 & $4.65 \%$ & \\
\hline & & Hu 2011 [12] & 13 & 0 & $0.00 \%$ & \\
\hline & \multirow[t]{17}{*}{ Pancreatitis } & Sato 2020 [24] & 30 & 1 & $3.33 \%$ & \multirow[t]{17}{*}{$4.04 \%$} \\
\hline & & Poley 2020 [25] & 41 & 0 & $0.00 \%$ & \\
\hline & & Tringali 2019 [26] & 18 & 1 & $5.56 \%$ & \\
\hline & & Lakhtakia 2019 [9] & 118 & 6 & $5.08 \%$ & \\
\hline & & Wu 2017 [6] & 32 & 2 & $6.25 \%$ & \\
\hline & & Schmidt 2017 [27] & 43 & 1 & $2.33 \%$ & \\
\hline & & Aepli 2017 [10] & 31 & 0 & $0.00 \%$ & \\
\hline & & Cote 2016 [1] & 57 & 3 & $5.26 \%$ & \\
\hline & & Chaput 2016 [28] & 92 & 5 & $5.43 \%$ & \\
\hline & & Walter 2015 [29] & 38 & 1 & $2.63 \%$ & \\
\hline & & Saxena 2015 [3] & 123 & 4 & $3.25 \%$ & \\
\hline & & $\mathrm{Hu} 2014$ [30] & 45 & 1 & $2.22 \%$ & \\
\hline & & Wagh 2013 [11] & 23 & 0 & $0.00 \%$ & \\
\hline & & Ryu 2013 [31] & 41 & 4 & $9.76 \%$ & \\
\hline & & Kahaleh 2013 [7] & 133 & 3 & $2.26 \%$ & \\
\hline & & Poley 2012 [32] & 23 & 0 & $0.00 \%$ & \\
\hline & & Moon 2012 [33] & 21 & 0 & $0.00 \%$ & \\
\hline
\end{tabular}


Table III. Cont.

\begin{tabular}{|c|c|c|c|c|c|c|}
\hline Stent & Adverse event & Study & Patient $(n)$ & Event $(n)$ & Incidence & Pooled date \\
\hline \multirow[t]{21}{*}{ FCSEMSS } & \multirow[t]{2}{*}{ Pancreatitis } & Park 2011 [34] & 43 & 6 & $13.95 \%$ & \multirow[t]{2}{*}{$4.04 \%$} \\
\hline & & $\mathrm{Hu} 2011$ [12] & 13 & 1 & $7.69 \%$ & \\
\hline & \multirow[t]{19}{*}{ Stent migration } & Sato 2020 [24] & 30 & 1 & $3.33 \%$ & \multirow[t]{19}{*}{$14.51 \%$} \\
\hline & & Poley 2020 [25] & 41 & 24 & $58.54 \%$ & \\
\hline & & Tringali 2019 [26] & 18 & 0 & $0.00 \%$ & \\
\hline & & Lakhtakia 2019 [9] & 118 & 5 & $4.24 \%$ & \\
\hline & & Wu 2017 [6] & 32 & 1 & $3.13 \%$ & \\
\hline & & Schmidt 2017 [27] & 43 & 2 & $4.65 \%$ & \\
\hline & & Aepli 2017 [10] & 31 & 1 & $3.23 \%$ & \\
\hline & & Cote 2016 [1] & 57 & 16 & $28.07 \%$ & \\
\hline & & Chaput 2016 [28] & 92 & 23 & $25.00 \%$ & \\
\hline & & Walter 2015 [29] & 38 & 11 & $28.95 \%$ & \\
\hline & & Saxena 2015 [3] & 123 & 12 & $9.76 \%$ & \\
\hline & & Hu 2014 [30] & 45 & 3 & $6.67 \%$ & \\
\hline & & Wagh 2013 [11] & 23 & 9 & $39.13 \%$ & \\
\hline & & Ryu 2013 [31] & 41 & 6 & $14.63 \%$ & \\
\hline & & Kahaleh 2013 [7] & 133 & 14 & $10.53 \%$ & \\
\hline & & Poley 2012 [32] & 23 & 1 & $4.35 \%$ & \\
\hline & & Moon 2012 [33] & 21 & 4 & $19.05 \%$ & \\
\hline & & Park 2011 [34] & 43 & 7 & $16.28 \%$ & \\
\hline & & Hu 2011 [12] & 13 & 0 & $0.00 \%$ & \\
\hline \multirow[t]{20}{*}{ MPSS } & \multirow[t]{5}{*}{ Abdominal pain } & Costamagna 2020 [8] & 154 & 2 & $1.30 \%$ & \multirow[t]{5}{*}{$4.71 \%$} \\
\hline & & Ohyama 2017 [35] & 10 & 0 & $0.00 \%$ & \\
\hline & & Canena 2014 [36] & 20 & 2 & $10.00 \%$ & \\
\hline & & Wu 2017 [6] & 37 & 0 & $0.00 \%$ & \\
\hline & & Cote 2016 [1] & 55 & 9 & $16.36 \%$ & \\
\hline & \multirow[t]{5}{*}{ Cholangitis } & Costamagna 2020 [8] & 154 & 34 & $22.08 \%$ & \multirow[t]{5}{*}{$15.94 \%$} \\
\hline & & Ohyama 2017 [35] & 10 & 1 & $10.00 \%$ & \\
\hline & & Canena 2014 [36] & 20 & 1 & $5.00 \%$ & \\
\hline & & Wu 2017 [6] & 37 & 7 & $18.92 \%$ & \\
\hline & & Cote 2016 [1] & 55 & 1 & $1.82 \%$ & \\
\hline & \multirow[t]{5}{*}{ Pancreatitis } & Costamagna 2020 [8] & 154 & 1 & $0.65 \%$ & \multirow[t]{5}{*}{$2.54 \%$} \\
\hline & & Ohyama 2017 [35] & 10 & 0 & $0.00 \%$ & \\
\hline & & Canena 2014 [36] & 20 & 0 & $0.00 \%$ & \\
\hline & & Wu 2017 [6] & 37 & 3 & $8.11 \%$ & \\
\hline & & Cote 2016 [1] & 55 & 3 & $5.45 \%$ & \\
\hline & \multirow[t]{5}{*}{ Stent migration } & Costamagna 2020 [8] & 154 & 1 & $0.65 \%$ & \multirow[t]{5}{*}{$6.52 \%$} \\
\hline & & Ohyama 2017 [35] & 10 & 0 & $0.00 \%$ & \\
\hline & & Canena 2014 [36] & 20 & 4 & $20.00 \%$ & \\
\hline & & Wu 2017 [6] & 37 & 3 & $8.11 \%$ & \\
\hline & & Cote 2016 [1] & 55 & 10 & $18.18 \%$ & \\
\hline
\end{tabular}


Table IV. Summary of rare adverse events

\begin{tabular}{|c|c|c|c|c|}
\hline Stent & Author & Patient $(n)$ & Adverse event $(n)$ & Incidence (\%) \\
\hline \multirow[t]{13}{*}{ BDBSs } & Basttistel 2020 [20] & 18 & Haemobilia (1) & \multirow{5}{*}{$\begin{array}{l}5.56 \\
6.67 \\
1.33 \\
0.67 \\
0.67\end{array}$} \\
\hline & \multirow[t]{4}{*}{ De Gregorio 2020 [19] } & \multirow[t]{4}{*}{150} & Haemobilia (10) & \\
\hline & & & Abdominal wall haematoma (2) & \\
\hline & & & Intestinal loop laceration (1) & \\
\hline & & & Pleural effusion (1) & \\
\hline & Siiki 2018 [15] & 6 & Null & 0 \\
\hline & Dopazo 2018 [5] & 10 & Liver abscess (1) & \multirow{4}{*}{$\begin{array}{c}10.00 \\
3.74 \\
15.89 \\
6.54\end{array}$} \\
\hline & \multirow[t]{3}{*}{ Mauri 2016 [21] } & \multirow[t]{3}{*}{107} & Haemobilia (4) & \\
\hline & & & Increased GGT/ALT (17) & \\
\hline & & & Biliary stone (7) & \\
\hline & Mauri 2015 [22] & 59 & Haemobilia (3) & \multirow{3}{*}{$\begin{array}{c}5.08 \\
7.69 \\
15.38\end{array}$} \\
\hline & \multirow[t]{2}{*}{ Giménez 2016 [23] } & \multirow[t]{2}{*}{13} & Haemobilia (1) & \\
\hline & & & Elevated ALP (2) & \\
\hline \multirow[t]{23}{*}{ FCSEMSS } & Sato 2020 [24] & 30 & Perforation (1) & 3.33 \\
\hline & \multirow[t]{3}{*}{ Poley 2020 [25] } & \multirow[t]{3}{*}{41} & Cholestasis (1) & \multirow{3}{*}{$\begin{array}{l}2.44 \\
2.44 \\
2.44\end{array}$} \\
\hline & & & Bleeding in bile duct (1) & \\
\hline & & & Elevated serum bilirubin (1) & \\
\hline & Tringali 2019 [26] & 18 & Null & 0 \\
\hline & \multirow[t]{4}{*}{ Lakhtakia 2019 [9] } & \multirow[t]{4}{*}{118} & Cholecystitis (3) & \multirow{4}{*}{$\begin{array}{l}2.54 \\
4.24 \\
2.54 \\
9.32\end{array}$} \\
\hline & & & Cholestasis (5) & \\
\hline & & & Cholelithiasis (3) & \\
\hline & & & Others (11) & \\
\hline & \multirow[t]{2}{*}{ Wu 2017 [6] } & \multirow[t]{2}{*}{32} & Bleeding (1) & \multirow{2}{*}{$\begin{array}{l}3.13 \\
6.25\end{array}$} \\
\hline & & & Sludge obstruction (2) & \\
\hline & \multirow[t]{3}{*}{ Schmidt 2017 [27] } & \multirow[t]{3}{*}{43} & Acute cholecystitis (1) & \multirow{3}{*}{$\begin{array}{c}2.33 \\
11.63 \\
2.33\end{array}$} \\
\hline & & & Stent occlusion (5) & \\
\hline & & & Sludge obstruction (1) & \\
\hline & Aepli 2017 [10] & 31 & Null & 0 \\
\hline & \multirow[t]{4}{*}{ Cote 2016 [1] } & \multirow[t]{4}{*}{57} & Bile duct obstruction (1) & \multirow{4}{*}{$\begin{array}{c}1.75 \\
1.75 \\
3.51 \\
15.79\end{array}$} \\
\hline & & & Jaundice (1) & \\
\hline & & & Secondary bile duct changes (2) & \\
\hline & & & Others (9) & \\
\hline & \multirow[t]{4}{*}{ Chaput 2016 [28] } & 92 & Haemorrhage (1) & 1.09 \\
\hline & & & Cholecystitis (1) & $\begin{array}{l}1.09 \\
1.09\end{array}$ \\
\hline & & & Liver abscess (1) & 4.35 \\
\hline & & & Biological abnormalities (4) & \\
\hline
\end{tabular}


Table IV. Cont.

\begin{tabular}{|c|c|c|c|c|}
\hline Stent & Author & Patient $(n)$ & Adverse event $(n)$ & Incidence (\%) \\
\hline \multirow[t]{26}{*}{ FCSEMSS } & \multirow[t]{6}{*}{ Walter 2015 [29] } & \multirow[t]{6}{*}{38} & Flare up of chronic pancreatitis (1) & \multirow{6}{*}{$\begin{array}{l}2.63 \\
2.63 \\
2.63 \\
2.63 \\
5.26 \\
2.63\end{array}$} \\
\hline & & & Cholecystitis (1) & \\
\hline & & & Portal vein thrombosis (1) & \\
\hline & & & Fever (1) & \\
\hline & & & Stent occlusion (2) & \\
\hline & & & Bleeding of duodenal varices (1) & \\
\hline & \multirow[t]{5}{*}{ Saxena 2015 [3] } & \multirow[t]{5}{*}{123} & Stent occlusion (6) & \multirow{5}{*}{$\begin{array}{l}4.88 \\
0.81 \\
0.81 \\
0.81 \\
0.81\end{array}$} \\
\hline & & & Tissue ingrowth (1) & \\
\hline & & & Stent fracture (1) & \\
\hline & & & Embedded stent (1) & \\
\hline & & & Stent-related death (1) & \\
\hline & $\mathrm{Hu} 2014$ [30] & 45 & Null & 0 \\
\hline & Wagh 2013 [11] & 23 & Bile duct stone (7) & 30.43 \\
\hline & Ryu 2013 [31] & 41 & Stent occlusion (2) & 4.88 \\
\hline & \multirow[t]{5}{*}{ Kahaleh 2013 [7] } & \multirow[t]{5}{*}{133} & Post-procedure pain (8) & 6.02 \\
\hline & & & Stent occlusion (4) & 3.01 \\
\hline & & & Bleeding (1) & 0.75 \\
\hline & & & Unravelling of the stent (2) & 1.50 \\
\hline & & & Hyperplastic reaction (1) & 0.75 \\
\hline & \multirow[t]{2}{*}{ Poley 2012 [32] } & \multirow[t]{2}{*}{23} & Cholecystitis (1) & \multirow{7}{*}{$\begin{array}{l}4.35 \\
8.70 \\
4.76 \\
9.30 \\
7.69 \\
7.69 \\
7.69\end{array}$} \\
\hline & & & Stent clogging (2) & \\
\hline & Moon 2012 [33] & 21 & Jaundice (1) & \\
\hline & Park 2011 [34] & 43 & Sludge (4) & \\
\hline & \multirow[t]{3}{*}{ Hu 2011 [12] } & \multirow[t]{3}{*}{13} & Continuing pyrexia (1) & \\
\hline & & & Abnormal liver function (1) & \\
\hline & & & Liver abscess (1) & \\
\hline \multirow[t]{12}{*}{ MPSS } & \multirow[t]{4}{*}{ Costamagna $2020[8]$} & \multirow[t]{4}{*}{154} & Post-endoscopic bleeding (5) & \multirow{4}{*}{$\begin{array}{l}3.25 \\
0.65 \\
5.84 \\
3.25\end{array}$} \\
\hline & & & Ileal perforation (1) & \\
\hline & & & Bile duct stone (9) & \\
\hline & & & Jaundice (5) & \\
\hline & Ohyama 2017 [35] & 10 & Null & 0 \\
\hline & Canena 2014 [36] & 20 & Stent clogging (2) & 10.00 \\
\hline & \multirow[t]{4}{*}{ Cote 2016 [1] } & \multirow[t]{4}{*}{55} & Bile duct obstruction (1) & \multirow{6}{*}{$\begin{array}{c}1.82 \\
1.82 \\
9.09 \\
12.73 \\
5.41 \\
8.11\end{array}$} \\
\hline & & & Jaundice (1) & \\
\hline & & & Secondary bile duct change (5) & \\
\hline & & & Others (7) & \\
\hline & \multirow[t]{2}{*}{ Wu 2017 [6] } & \multirow[t]{2}{*}{37} & Post-sphincterotomy bleeding (2) & \\
\hline & & & Sludge impaction (3) & \\
\hline
\end{tabular}

GGT - $\gamma$-glutamyl transpeptidase, ALT - alanine transaminase, ALP - alkaline phosphatase. 
Study

\section{BDBSs}

Battistel 2020

De Gregorio 2020

Siiki 2018

Dopazo 2018

Mauri 2016

Mauri 2015

Giménez 2016

Subtotal $\left(I^{2}=92.71 \%, p<0.001\right)$

\section{FCSEMSS}

Sato 2020

Poley 2020

Tringali 2019

Lakhtakia 2019

Wu 2017

Schmidt 2017

Aepli 2017

Cote 2016

Chaput 2016

Walter 2015

Saxena 2015

$\mathrm{Hu} 2014$

Wagh 2013

Ryu 2013

Kahaleh 2013

Poley 2012

Moon 2012

Park 2011

Hu 2011

Subtotal $\left(l^{2}=88.28 \%, p<0.001\right)$

\section{MPSs}

Costamagna 2020

Ohyama 2017

Canena 2014

Wu 2017

Cote 2016

Subtotal $\left(I^{2}=76.54 \%, p<0.001\right)$

Heterogeneity between groups: $p=0.558$

Overall $\left(I^{2}=89.28 \%, p<0.001\right)$
ES $(95 \% \mathrm{Cl})$

Weight (\%)
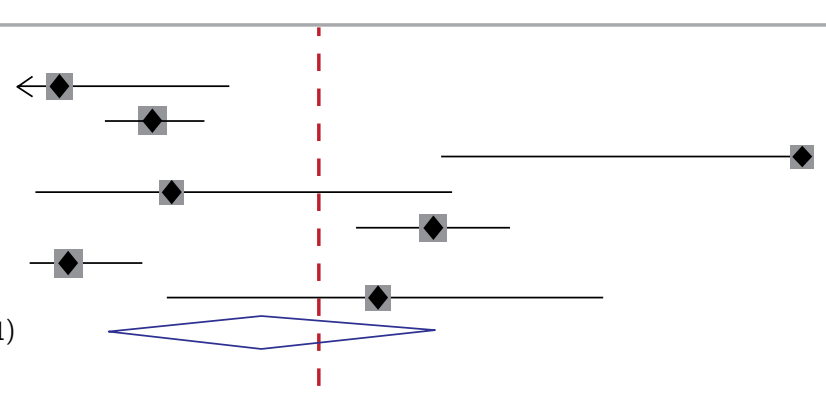

$0.06(0.00,0.27) \quad 2.9$

$0.17(0.12,0.24) \quad 3.73$

$1.00(0.54,1.00) \quad 2.00$

$0.20(0.03,0.56) \quad 2.45$

$0.53(0.43,0.63) \quad 3.67$

$0.07(0.02,0.16) \quad 3.52$

$0.46(0.19,0.75) \quad 2.67$

$0.31(0.12,0.54) \quad 20.94$

$0.27(0.12,0.46) \quad 3.23$

$0.41(0.26,0.58) \quad 3.38$

$0.39(0.17,0.64) \quad 2.91$

$0.51(0.41,0.60) \quad 3.69$

$0.31(0.16,0.50) \quad 3.26$

$0.35(0.21,0.51) \quad 3.40$

$0.10(0.02,0.26) \quad 3.25$

$0.74(0.60,0.84) \quad 3.51$

$0.49(0.38,0.60) \quad 3.64$

$0.50(0.33,0.67) \quad 3.35$

$0.25(0.18,0.34) \quad 3.70$

$0.11(0.04,0.24) \quad 3.42$

$0.74(0.52,0.90) \quad 3.08$

$0.37(0.22,0.53) \quad 3.38$

$0.25(0.18,0.33) \quad 3.71$

$0.96(0.78,1.00) \quad 3.08$

$0.24(0.08,0.47) \quad 3.02$

$0.44(0.29,0.60) \quad 3.40$

$0.31(0.09,0.61) \quad 2.67$

$0.40(0.31,0.50) \quad 63.06$

$0.38(0.30,0.46) \quad 3.73$

$0.20(0.03,0.56) \quad 2.45$

$0.45(0.23,0.68) \quad 2.99$

$0.49(0.32,0.66) \quad 3.33$

$0.67(0.53,0.79) \quad 3.49$

$0.46(0.31,0.61) \quad 16.00$

$0.39(0.31,0.47) \quad 100.00$

\begin{tabular}{cccc|c}
\hline & 1 & 1 & 1 \\
0.25 & 0.50 & 0.75 & 1.00
\end{tabular}

Figure 4. Forest plot comparing adverse events rate in different groups using effect size (ES) with ordinary weighting

ES - effect size, $\mathrm{Cl}$ - confidence interval.

$83.86 \%$ and $p<0.001$ for FCSEMSs, $I^{2}=85.57 \%$ and $p<0.001$ for MPSs)

\section{Pancreatitis}

All 31 included studies reported pancreatitis. After the meta-analysis in Figure 8, the lowest rate was found in the BDBS group (0.00, 95\% Cl: $0.00-$ 0.01 ). The pooled rate in the MPS group was 0.02 (95\% Cl: 0.00-0.06), lower than that in the FCSEMS group (0.03, 95\% Cl: 0.02-0.05). The heterogeneity was low for all 3 groups $\left(R^{2}=35.57 \%\right.$ and $p=0.16$ for BDBSs, $I^{2}=13.57 \%$ and $p=0.29$ for FCSEMSs, $R^{2}=48.72 \%$ and $p=0.10$ for MPSs). 
Subtotal $\left(I^{2}=69.39 \%, p<0.001\right)$

\section{FCSEMSS}

Sato 2020

Poley 2020

Tringali 2019

Lakhtakia 2019

Wu 2017

Schmidt 2017

Aepli 2017

Cote 2016

Chaput 2016

Walter 2015

Saxena 2015

Hu 2014

Wagh 2013

Ryu 2013

Kahaleh 2013

Poley 2012

Moon 2012

Park 2011

Hu 2011

Subtotal $\left(I^{2}=78.47 \%, p<0.001\right)$

\section{MPSs}

Costamagna 2020

Ohyama 2017

Canena 2014

Wu 2017

Cote 2016

Subtotal $\left(I^{2}=78.44 \%, p<0.001\right)$

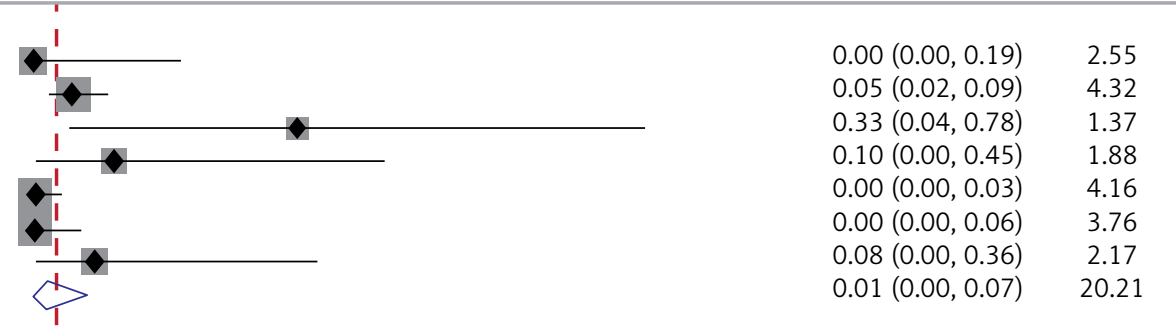

Heterogeneity between groups: $p=0.937$

Overall $\left(I^{2}=75.87 \%, p<0.001\right)$

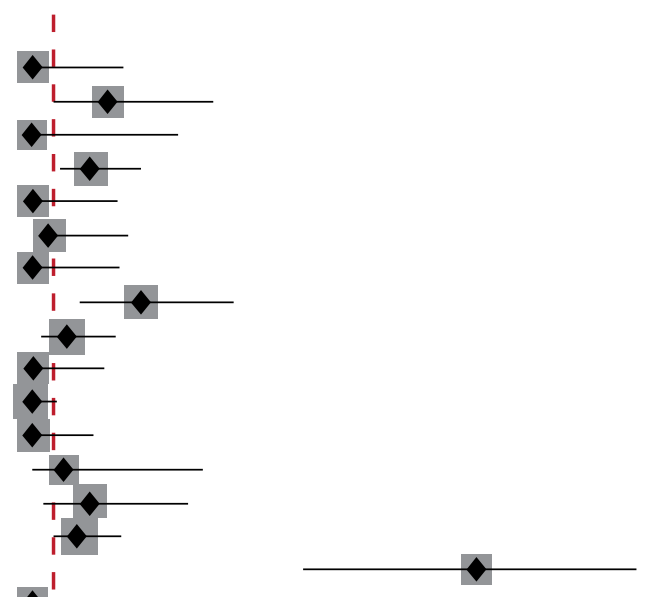

$0.00(0.00,0.12) \quad 3.12$

$0.10(0.03,0.23) \quad 3.44$

$0.00(0.00,0.19) \quad 2.55$

$0.08(0.04,0.14) \quad 4.21$

$0.00(0.00,0.11) \quad 3.19$

$0.02(0.00,0.12) \quad 3.49$

$0.00(0.00,0.11) \quad 3.16$

$0.14(0.06,0.26) \quad 3.73$

$0.04(0.01,0.11) \quad 4.07$

$0.00(0.00,0.09) \quad 3.37$

$0.00(0.00,0.03) \quad 4.23$

$0.00(0.00,0.08) \quad 3.53$

$0.04(0.00,0.22) \quad 2.83$

$0.07(0.02,0.20) \quad 3.44$

$0.06(0.03,0.12) \quad 4.27$

$0.57(0.34,0.77) \quad 2.83$

$0.00(0.00,0.16) \quad 2.73$

$0.00(0.00,0.08) \quad 3.49$

$0.00(0.00,0.25) \quad 2.17$

$0.03(0.01,0.06) \quad 63.86$

$0.01(0.00,0.05) \quad 4.33$

$0.00(0.00,0.31) \quad 1.88$

$0.10(0.01,0.32) \quad 2.67$

$0.00(0.00,0.09) \quad 3.34$

$0.16(0.08,0.29) \quad 3.70$

$0.03(0.00,0.12) \quad 15.93$

$0.03(0.01,0.05) \quad 100.00$

\begin{tabular}{cccc|cc}
\hline & & & \\
0.25 & 0.50 & 0.75 & 1.00
\end{tabular}

Figure 5. Forest plot comparing abdominal pain rate in different groups using effect size (ES) with ordinary weighting

ES - effect size, $\mathrm{Cl}$ - confidence interval.

\section{Technical success}

All 31 included studies reported technical success. From the result of this meta-analysis, technical success was most likely to achieve when using BDBS $(1.00,95 \% \mathrm{Cl}: 1.00-1.00)$. The pooled technical success rate of MPS was 0.95 (95\% Cl: $0.88-0.99$ ), which was higher than that of FCSEMS $(0.90,95 \% \mathrm{Cl}$ : 0.85-0.94). The level of heterogeneity was low for the BDBS group $\left(I^{2}=0.00 \%\right.$ and $\left.p=0.62\right)$, and high for the other 2 groups $\left(I^{2}=76.72 \%\right.$ and $p<$ 0.001 for FCSEMSs, $R^{2}=59.05 \%$ and $p=0.04$ for MPSs) (Figure 9).

\section{Treatment success}

All 31 included studies reported treatment success. From the result of this meta-analysis, treat- 


\section{BDBSs}

Battistel 2020

De Gregorio 2020

Siiki 2018

Dopazo 2018

Mauri 2016

Mauri 2015

Giménez 2016

Subtotal $\left(l^{2}=92.01 \%, p<0.001\right)$

\section{FCSEMSS}

Sato 2020

Poley 2020

Tringali 2019

Lakhtakia 2019

Wu 2017

Schmidt 2017

Aepli 2017

Cote 2016

Chaput 2016

Walter 2015

Saxena 2015

Hu 2014

Wagh 2013

Ryu 2013

Kahaleh 2013

Poley 2012

Moon 2012

Park 2011

Hu 2011

Subtotal $\left(I^{2}=77.63 \%, p<0.001\right)$

MPSs

Costamagna 2020

Ohyama 2017

Canena 2014

Wu 2017

Cote 2016

Subtotal $\left(I^{2}=79.74 \%, p<0.001\right)$

Heterogeneity between groups: $p=0.60$

Overall $\left(I^{2}=84.96 \%, p<0.001\right)$

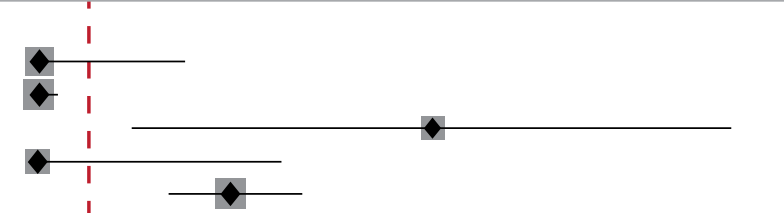

$0.00(0.00,0.19) \quad 2.79$

$0.00(0.00,0.02) \quad 3.92$

$0.50(0.12,0.88) \quad 1.74$

$0.00(0.00,0.31) \quad 2.23$

$0.24(0.17,0.34) \quad 3.83$

$0.00(0.00,0.06) \quad 3.61$

$0.15(0.02,0.45) \quad 2.49$

$0.05(0.00,0.20) \quad 20.61$

$0.17(0.06,0.35) \quad 3.21$

$0.24(0.12,0.40) \quad 3.41$

$0.33(0.13,0.59) \quad 2.79$

$0.15(0.09,0.23) \quad 3.86$

$0.13(0.04,0.29) \quad 3.25$

$0.02(0.00,0.12) \quad 3.44$

$0.06(0.01,0.21) \quad 3.23$

$0.04(0.00,0.12) \quad 3.59$

$0.07(0.02,0.14) \quad 3.79$

$0.13(0.04,0.28) \quad 3.37$

$0.04(0.01,0.09) \quad 3.87$

$0.02(0.00,0.12) \quad 3.47$

$0.00(0.00,0.15) \quad 3.00$

$0.00(0.00,0.09) \quad 3.41$

$0.00(0.00,0.03) \quad 3.89$

$0.13(0.03,0.34) \quad 3.00$

$0.00(0.00,0.16) \quad 2.93$

$0.05(0.01,0.16) \quad 3.44$

$0.00(0.00,0.25) \quad 2.49$

$0.06(0.03,0.10) \quad 63.43$

$0.22(0.16,0.29) \quad 3.93$

$0.10(0.00,0.45) \quad 2.23$

$0.05(0.00,0.25) \quad 2.88$

$0.19(0.08,0.35) \quad 3.35$

$0.02(0.00,0.10) \quad 3.57$

$0.11(0.02,0.23) \quad 15.96$

$0.06(0.03,0.10) \quad 100.00$

\begin{tabular}{ccccc}
1 & 1 & 1 & \\
\hline & 0.25 & 0.50 & 0.75 & 1.00
\end{tabular}

Figure 6. Forest plot comparing cholangitis rate in different groups using effect size (ES) with ordinary weighting

ES - effect size, $\mathrm{Cl}$ - confidence interval.

ment success was most likely to be achieved when using BDBS (1.00, 95\% Cl: 1.00-1.00). The pooled treatment success rate of MPSs was $0.88(95 \% \mathrm{Cl}$ : 0.72-0.98), which was higher than that of FCSEMSs $(0.82,95 \% \mathrm{Cl}: 0.76-0.87)$. The level of heterogeneity was low for the BDBS group $\left(I^{2}=0.00 \%\right.$ and $\left.p=0.69\right)$ and high for the other 2 groups $\left(F^{2}=77.62 \%\right.$ and $p<0.001$ for FCSEMSs, $I^{2}=87.32 \%$ and $p<0.001$ for MPSs) (Figure 10).

\section{Stricture recurrent}

All 31 included studies reported recurrent stricture. From the result of this meta-analysis, stricture recurrence was least likely to occur in the MPS group $(0.07,95 \% \mathrm{Cl}: 0.03-0.13)$. The pooled stricture recurrent rate of FCSEMSs was 0.11 (95\% Cl: 0.08-0.15), lower than that of BDBSs group (0.21, $95 \% \mathrm{Cl}: 0.16-$ $0.26)$. The heterogeneity was evaluated as low for all 3 groups $\left(I^{2}=11.59 \%\right.$ and $p=0.34$ for BDBSs, 
Study

\section{BDBSs}

Battistel 2020

De Gregorio 2020

Siiki 2018

Dopazo 2018

Mauri 2016

Mauri 2015

Giménez 2016

Subtotal $\left(I^{2}=0.00 \%, p=0.99\right)$

\section{FCSEMSS}

Sato 2020

Poley 2020

Tringali 2019

Lakhtakia 2019

Wu 2017

Schmidt 2017

Aepli 2017

Cote 2016

Chaput 2016

Walter 2015

Saxena 2015

Hu 2014

Wagh 2013

Ryu 2013

Kahaleh 2013

Poley 2012

Moon 2012

Park 2011

Hu 2011

Subtotal $\left(I^{2}=83.86 \%, p<0.001\right)$

\section{MPSs}

Costamagna 2020

Ohyama 2017

Canena 2014

Wu 2017

Cote 2016

Subtotal $\left(I^{2}=85.57 \%, p<0.001\right)$
$0.00(0.00,0.19) \quad 2.78$

$0.03(0.01,0.08) \quad 3.94$

$0.00(0.00,0.46) \quad 1.71$

$0.00(0.00,0.31) \quad 2.21$

$0.02(0.00,0.07) \quad 3.85$

$0.02(0.00,0.09) \quad 3.62$

$0.00(0.00,0.25) \quad 2.47$

$0.01(0.00,0.02) \quad 20.58$

$0.03(0.00,0.17) \quad 3.20$

$0.59(0.42,0.74) \quad 3.41$

$0.00(0.00,0.19) \quad 2.78$

$0.04(0.01,0.10) \quad 3.88$

$0.03(0.00,0.16) \quad 3.25$

$0.05(0.01,0.16) \quad 3.44$

$0.03(0.00,0.17) \quad 3.23$

$0.28(0.17,0.42) \quad 3.60$

$0.25(0.17,0.35) \quad 3.80$

$0.29(0.15,0.46) \quad 3.37$

$0.10(0.05,0.16) \quad 3.89$

$0.07(0.01,0.18) \quad 3.47$

$0.39(0.20,0.61) \quad 2.99$

$0.15(0.06,0.29) \quad 3.41$

$0.11(0.06,0.17) \quad 3.91$

$0.04(0.00,0.22) \quad 2.99$

$0.19(0.05,0.42) \quad 2.91$

$0.16(0.07,0.31) \quad 3.44$

$0.00(0.00,0.25) \quad 2.47$

$0.12(0.07,0.19) \quad 63.46$

$0.01(0.00,0.04) \quad 3.95$

$0.00(0.00,0.31) \quad 2.21$

$0.20(0.06,0.44) \quad 2.87$

$0.08(0.02,0.22) \quad 3.35$

$0.18(0.09,0.31) \quad 3.58$

$0.07(0.00,0.20) \quad 15.96$

$0.08(0.05,0.13) \quad 100.00$

Heterogeneity between groups: $p<0.001$

Overall $\left(I^{2}=84.46 \%, p<0.001\right)$

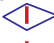

\begin{tabular}{ccccc}
1 & 1 & 1 & \\
\hline & 0.25 & 0.50 & 0.75 & 1.00
\end{tabular}

Figure 7. Forest plot comparing stent migration rate in different groups using effect size (ES) with ordinary weighting

ES - effect size, $\mathrm{Cl}$ - confidence interval.

$R^{2}=38.85 \%$ and $p=0.05$ for FCSEMSs, $R^{2}=39.30 \%$ and $p=0.16$ for MPSs) (Figure 11).

\section{Intervention frequency and recurrent time}

In this meta-analysis, the cumulative need for intervention was reported in all 31 studies (Table V). For BDBSs, only 1 intervention of implantation was required to achieve clinical success, without the need for removal. For FCSEMSs, 1 implantation followed by 1 removal was required, as planned. However, for patients with persistent stricture during follow-up, ERCP was required 6 months after initial implantation, to replace FCSEMSs. Therefore, the cumulative need of intervention for FCSEMSs ranged from 2 to 3. Compared with the above 2 stents, the most obvious drawback of MPSs was the repeated needs of interventions. As reported in 5 of the included studies, its cumulate need of intervention ranged from 2 to 6. On the other hand, 21 of the included studies re- 


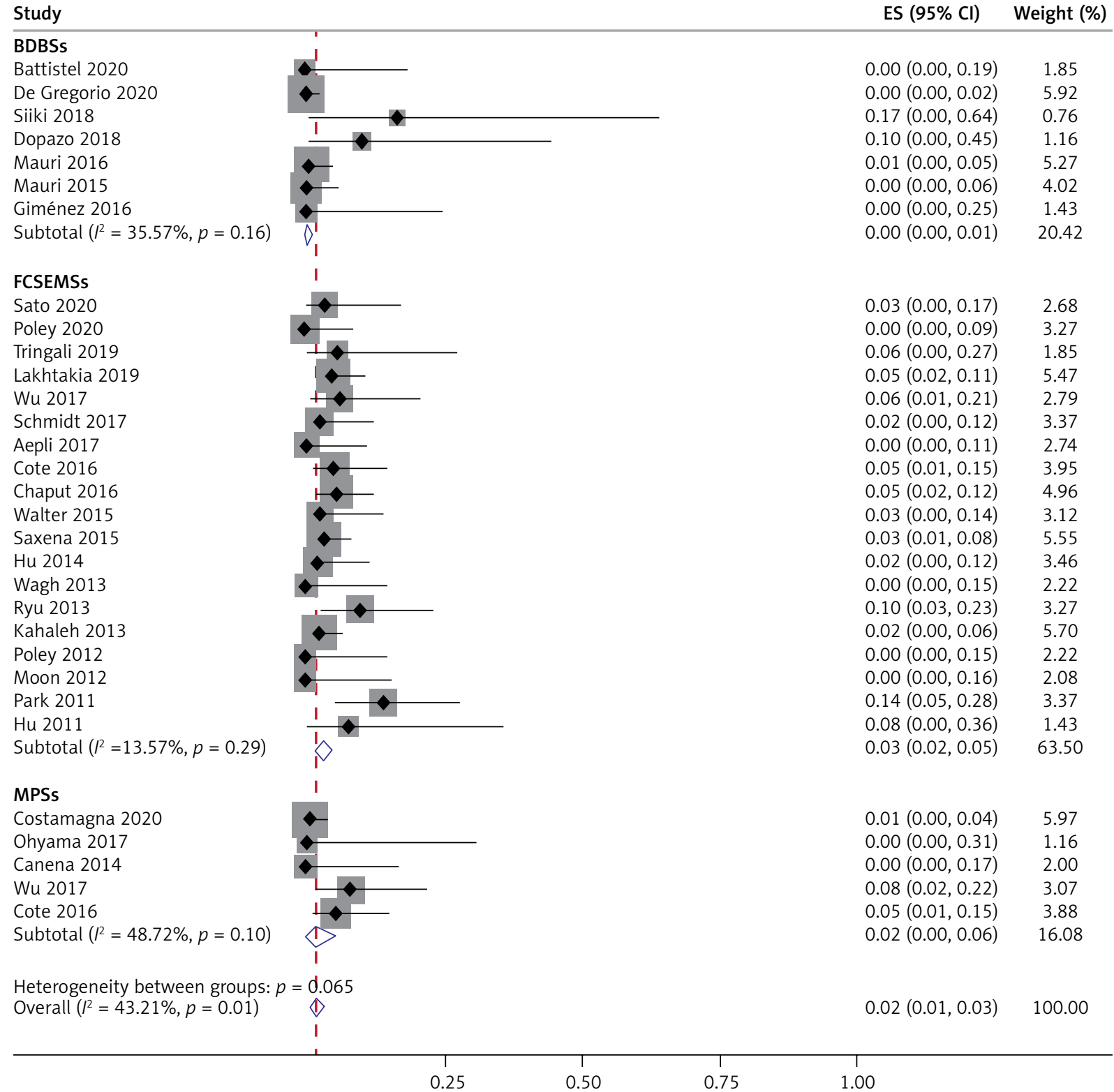

Figure 8. Forest plot comparing pancreatitis rate in different groups using effect size (ES) with ordinary weighting

ES - effect size, $\mathrm{Cl}$ - confidence interval.

ported the duration from treatment success to stricture recurrent (Table V). BDBSs had the longest duration, at 16.1 months, and the duration for FCSEMSs was 7.0 months, longer than for MPSs (6.6 months).

\section{Discussion}

This meta-analysis was conducted to compare the therapeutic efficacy of 3 common stents (BDBSs, FCSEMSs, and MPSs) in endoscopic treatment of BBS, and the main goal was to draw a conclusion about which kind of stents should be recommended. According to the results of our study, BDBSs had the highest clinical success rate, which was associated with the easiest achievement of technical success. On the one hand, BDBSs were implanted in the biliary tract through a single intervention, without needing to consider the risk during stent removal $[16,21]$. On the other hand, owing to the property of self-expansion, BDBSs could be initially loaded in 


\section{BDBSs}

Battistel 2020

De Gregorio 2020

Siiki 2018

Dopazo 2018

Mauri 2016

Mauri 2015

Giménez 2016

Subtotal $\left(I^{2}=0.00 \%, p=0.62\right)$

\section{FCSEMSS}

Sato 2020

Poley 2020

Tringali 2019

Lakhtakia 2019

Wu 2017

Schmidt 2017

Aepli 2017

Cote 2016

Chaput 2016

Saxena 2015

Hu 2014

Wagh 2013

Kahaleh 2013

Poley 2012

Moon 2012

Park 2011

Hu 2011

Subtotal $\left(I^{2}=76.62 \%, p<0.001\right)$

\section{MPSs}

Costamagna 2020

Ohyama 2017

Canena 2014

Wu 2017

Cote 2016

Subtotal $\left(I^{2}=59.05 \%, p=0.04\right)$

eity between groups: $p<0.00$

Overall $\left({ }^{2}=82.61 \%, p<0.001\right.$

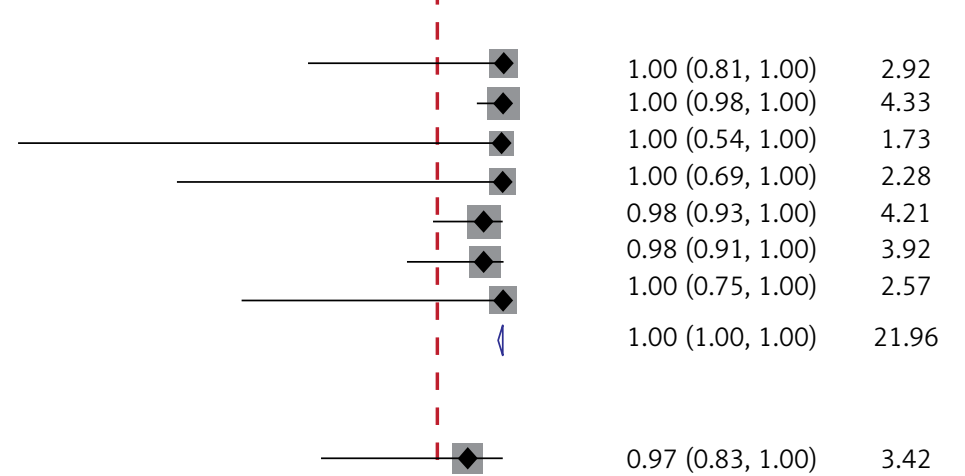

$0.80(0.65,0.91) \quad 3.67$

$0.83(0.59,0.96) \quad 2.92$

$0.92(0.85,0.96) \quad 4.25$

$0.97(0.84,1.00) \quad 3.47$

$0.67(0.51,0.81) \quad 3.71$

$0.94(0.79,0.99) \quad 3.45$

$0.95(0.85,0.99) \quad 3.90$

$0.78(0.68,0.86) \quad 4.15$

$0.89(0.82,0.94) \quad 4.26$

$0.78(0.63,0.89) \quad 3.74$

$1.00(0.85,1.00) \quad 3.17$

$0.74(0.65,0.81) \quad 4.29$

$1.00(0.85,1.00) \quad 3.17$

$1.00(0.84,1.00) \quad 3.08$

$0.91(0.78,0.97) \quad 3.71$

$0.92(0.64,1.00) \quad 2.57$

$0.90(0.85,0.94) \quad 60.93$

$0.97(0.93,0.99) \quad 4.33$

$0.80(0.44,0.97) \quad 2.28$

$1.00(0.83,1.00) \quad 3.03$

$0.95(0.82,0.99) \quad 3.59$

$0.87(0.76,0.95) \quad 3.88$

$0.95(0.88,0.99) \quad 17.11$

$0.94(0.90,0.97) \quad 100.00$

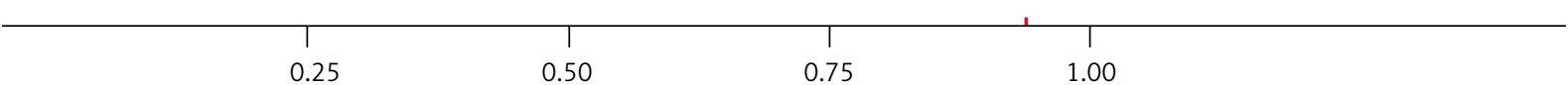

Figure 9. Forest plot comparing technical success rate in different groups using effect size (ES) with ordinary weighting

ES - effect size, $\mathrm{Cl}$ - confidence interval.

a thin delivery catheter (6-15 F) and thus were able to pass through the tight stricture without any difficulties $[16,21]$. The highest clinical success rate of BDBSs was also attributed to the relatively low migration rate. With the development of biodegrada- ble materials (such as polydioxanone) for fabricating BDBSs, this kind of stent does not require a silicone covering on its surface, which was the main reason for the high migration rate of FCSEMSs. However, the stricture recurrence rate of BDBSs was the highest 


\section{BDBSs}

Battistel 2020

De Gregorio 2020

Siiki 2018

Dopazo 2018

Mauri 2016

Mauri 2015

Giménez 2016

Subtotal $\left(I^{2}=0.00 \%, p=0.69\right)$

\section{FCSEMSS}

Sato 2020

Poley 2020

Tringali 2019

Lakhtakia 2019

Wu 2017

Schmidt 2017

Aepli 2017

Cote 2016

Chaput 2016

Walter 2015

Saxena 2015

Hu 2014

Wagh 2013

Ryu 2013

Kahaleh 2013

Moon 2012

Park 2011

Hu 2011

Subtotal $\left(R^{2}=77.62 \%, p<0.001\right)$

\section{MPSs}

Costamagna 2020

Ohyama 2017

Canena 2014

Wu 2017

Cote 2016

Subtotal $\left(I^{2}=87.32 \%, p<0.001\right)$

Heterogeneity between groups $p<0.001$

Overall $\left(I^{2}=89.86 \%, p<0.001\right)$

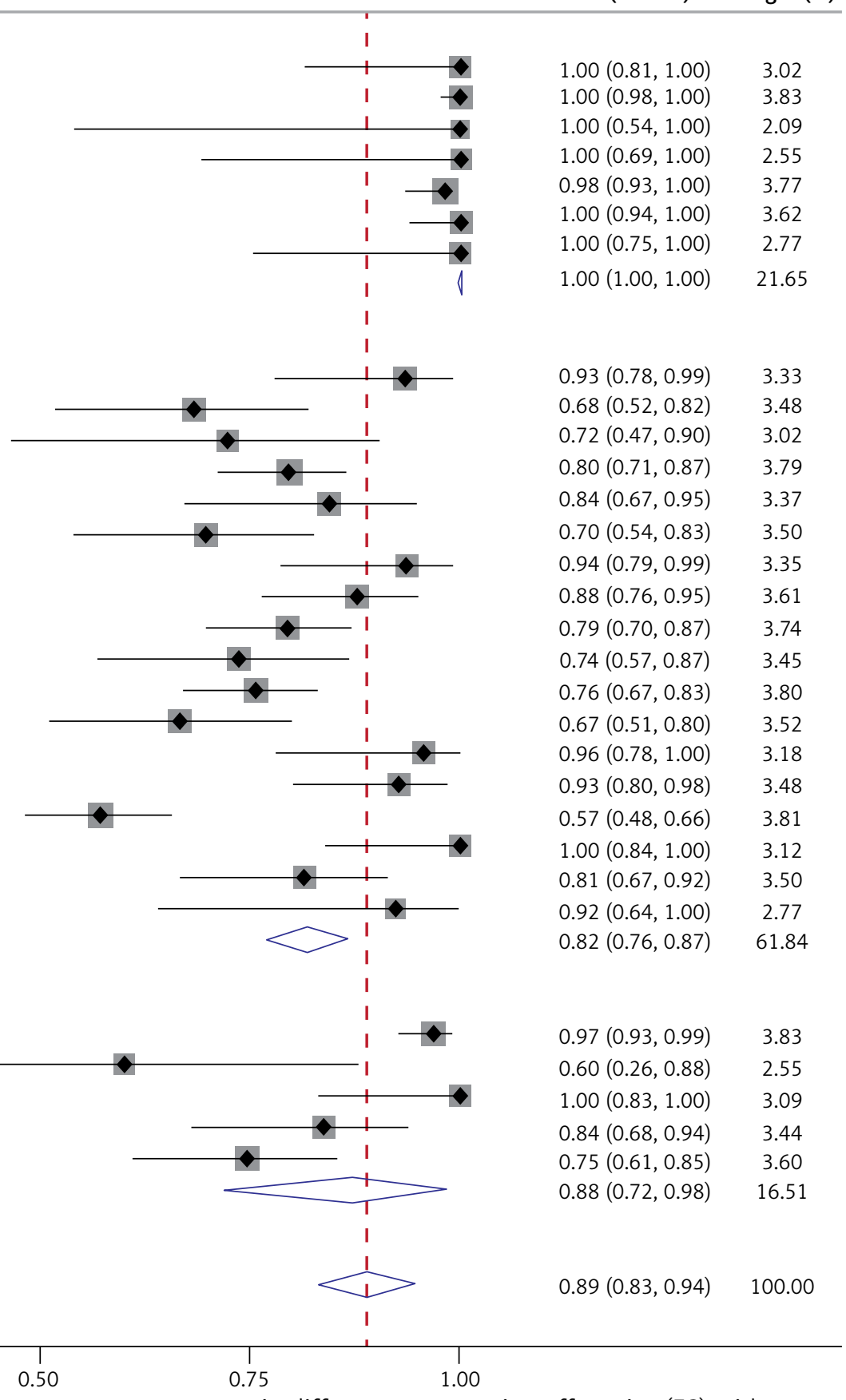

0.25
$1.00(0.98,1.00) \quad 3.83$

$1.00(0.75,1.00) \quad 2.77$

$1.00(1.00,1.00) \quad 21.65$

$0.93(0.78,0.99) \quad 3.33$

$0.68(0.52,0.82) \quad 3.48$

$0.72(0.47,0.90) \quad 3.02$

$0.84(0.67,0.95) \quad 3.37$

$0.70(0.54,0.83) \quad 3.50$

$0.94(0.79,0.99) \quad 3.35$

$0.88(0.76,0.95) \quad 3.61$

(0.70, 0.87)

$0.67(0.51,0.80) \quad 3.52$

$0.93(0.80,0.98) \quad 3.48$

$0.57(0.48,0.66) \quad 3.81$

$1.00(0.84,1.00)$

$0.92(0.64,1.00) \quad 2.77$

.84

83

$0.60(0.26,0.88) \quad 2.55$

$1.00(0.83,1.00) \quad 3.09$

$0.84(0.68,0.94) \quad 3.44$

$0.75(0.61,0.85) \quad 3.60$

$0.88(0.72,0.98) \quad 16.51$

$89(0.83,0.94) \quad 100.00$
$0.96(0.78,1.00) \quad 3.18$

\begin{tabular}{|c|c|c|c|}
\hline & $\mid$ & 1 & \\
0.25 & 0.50 & 0.75 & 1.00
\end{tabular}

Figure 10. Forest plot comparing treatment success rate in different groups using effect size (ES) with ordinary weighting

ES - effect size, $\mathrm{Cl}$ - confidence interval.

because of the uncontrolled degradation rate. BDBSs were mainly composed of amorphous regions of the matrix and crystalline area of the polymer, and the latter determined the mechanical and physical properties of these stents $[16,23]$. The degradation of the crystalline area in vivo was influenced by many factors, so it was difficult to control the effective duration of these stents. As reported by Siiki et al., the stents in 1 patient were invisible at 3 months, but in another they were still in place at 6 months [15]. 
Study

\section{BDBSs}

Battistel 2020

De Gregorio 2020

Siiki 2018

Dopazo 2018

Mauri 2016

Mauri 2015

Giménez 2016

Subtotal $\left(I^{2}=11.59 \%, p=0.34\right)$

\section{FCSEMSS}

Sato 2020

Poley 2020

Tringali 2019

Lakhtakia 2019

Wu 2017

Schmidt 2017

Aepli 2017

Cote 2016

Chaput 2016

Walter 2015

Saxena 2015

Hu 2014

Wagh 2013

Ryu 2013

Moon 2012

Park 2011

Hu 2011

Subtotal $\left(R^{2}=38.85 \%, p=0.05\right)$

\section{MPSs}

Costamagna 2020

Ohyama 2017

Canena 2014

Wu 2017

Cote 2016

Subtotal $\left(R^{2}=39.30 \%, p=0.16\right)$

Heterogeneity between groups: $p<0.001$

Overall $\left(I^{2}=59.29 \%, p<0.001\right)$

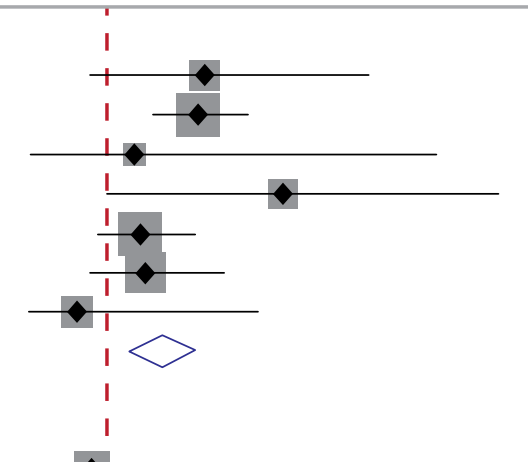

$0.28(0.10,0.53) \quad 2.36$

$0.27(0.20,0.34) \quad 5.45$

$0.17(0.00,0.64) \quad 1.08$

$0.40(0.12,0.74) \quad 1.58$

$0.18(0.11,0.26) \quad 5.08$

$0.19(0.10,0.31) \quad 4.26$

$0.08(0.00,0.36) \quad 1.91$

$0.21(0.16,0.26) \quad 21.71$

$0.10(0.02,0.27) \quad 3.17$

$0.17(0.07,0.32) \quad 3.68$

$0.11(0.01,0.35) \quad 2.36$

$0.16(0.10,0.24) \quad 5.19$

$0.09(0.02,0.25) \quad 3.27$

$0.05(0.01,0.16) \quad 3.76$

$0.23(0.10,0.41) \quad 3.22$

$0.12(0.05,0.24) \quad 4.20$

$0.17(0.10,0.27) \quad 4.89$

$0.16(0.06,0.31) \quad 3.55$

$0.04(0.01,0.09) \quad 5.24$

$0.04(0.01,0.15) \quad 3.83$

$0.13(0.03,0.34) \quad 2.74$

$0.15(0.06,0.29) \quad 3.68$

$0.05(0.00,0.24) \quad 2.60$

$0.16(0.07,0.31) \quad 3.76$

$0.08(0.00,0.36) \quad 1.91$

$0.11(0.08,0.15) \quad 61.05$

$0.08(0.04,0.13) \quad 5.48$

$0.00(0.00,0.31) \quad 1.58$

$0.20(0.06,0.44) \quad 2.52$

$0.14(0.05,0.29) \quad 3.51$

$0.04(0.00,0.13) \quad 4.15$

$0.07(0.03,0.13) \quad 17.24$

$0.12(0.09,0.16) \quad 100.00$

\begin{tabular}{ccc}
\hline & 1 & 1 \\
-0.5 & 0 & 0.5
\end{tabular}

Figure 11. Forest plot comparing stricture recurrent rate in different groups using effect size (ES) with ordinary weighting

ES - effect size, Cl - confidence interval.

Comparisons between MPSs and FCSEMSs have been conducted in several previous studies. In a meta-analysis performed by Qin et al., they found that FCSEMSs had a lower clinical success rate than MPSs $(\mathrm{OR}=0.48)[14]$. However, the finding was opposite in another meta-analysis performed by Siiki et al. [39]. They showed that the clinical success rate of FCSMESs was higher than for MPSs (0.77 vs. 0.33). In our meta-analysis, the results demonstrated that MPSs had better clinical outcome than FCSEMSs 
Table V. Summary of intervention number and recurrence time

\begin{tabular}{|c|c|c|c|}
\hline Stent & Study & $\begin{array}{l}\text { Intervention number } \\
\text { (mean) } n\end{array}$ & $\begin{array}{l}\text { Recurrent time } \\
\text { (mean) [months] }\end{array}$ \\
\hline \multirow[t]{7}{*}{ BDBSs } & Battistel 2020 [20] & 1 & NM \\
\hline & De Gregorio 2020 [19] & 1 & 27.8 \\
\hline & Siiki 2018 [15] & 1 & 17.0 \\
\hline & Dopazo 2018 [5] & 1 & 9.0 \\
\hline & Mauri 2016 [21] & 1 & 15.4 \\
\hline & Mauri 2015 [22] & 1 & 16.2 \\
\hline & Giménez 2016 [23] & 1 & 11.0 \\
\hline \multirow[t]{19}{*}{ FCSEMSS } & Sato 2020 [24] & 2 & 19.5 \\
\hline & Poley 2020 [25] & 2 & 3.4 \\
\hline & Tringali 2019 [26] & 2 & 7.9 \\
\hline & Lakhtakia 2019 [9] & 2 & NM \\
\hline & Wu 2017 [6] & 2 & $12.7^{\#}$ \\
\hline & Schmidt 2017 [27] & 2 & NM \\
\hline & Aepli 2017 [10] & 2 & 12.8 \\
\hline & Cote 2016 [1] & 2.14 & NM \\
\hline & Chaput 2016 [28] & 2 & 4.2 \\
\hline & Walter 2015 [29] & 2 & $4.5^{\#}$ \\
\hline & Saxena 2015 [3] & 2.2 & 4.0 \\
\hline & Hu 2014 [30] & 2 & 3.0 \\
\hline & Wagh 2013 [11] & 2.4 & NM \\
\hline & Ryu 2013 [31] & 2 & NM \\
\hline & Kahaleh 2013 [7] & 2 & NM \\
\hline & Poley 2012 [32] & 2 & NM \\
\hline & Moon 2012 [33] & 2 & 1.5 \\
\hline & Park 2011 [34] & 2 & NM \\
\hline & Hu 2011 [12] & 2 & 3.0 \\
\hline \multirow[t]{5}{*}{ MPSS } & Costamagna 2020 [8] & 4.2 & 1.5 \\
\hline & Ohyama 2017 [35] & 5.1 & - \\
\hline & Canena 2014 [36] & $5.5^{\#}$ & 11.5 \\
\hline & Wu 2017 [6] & $2^{\#}$ & $13.5^{\#}$ \\
\hline & Cote 2016 [1] & 3.24 & NM \\
\hline
\end{tabular}

(0.69 vs. 0.67). The reason for these contradicted results might be that each study aimed at a different range of patients with various causes of biliary stricture. Generally, MPSs tended to be applied in BBS caused by cholecystectomy, which had a good prognosis after endoscopic treatment. However, FCSEMSs were more often applied in BBS with poor prognosis, such as CP and LT $[39,40]$.
The total adverse event rates for these 3 stents were similar (0.31 for BDBSs, 0.40 for FCSEMSs, and 0.46 for MPSs), but the most common event related to each stent differed. For BDBSs, the most common adverse event was cholangitis. The reason is still unknown, but the reject reaction in the common bile duct caused by stent fragments after hydrolysis might be one of the factors [41]. Abdom- 
inal pain was the secondary common adverse event of BDBSs, owing to the forceful radial expansion of these stents. For FCSEMSs, stent migration was the most common adverse event. These metal stents were deliberately covered with silicone to make removal easy, but this also caused the stents to easily slip from the biliary tract $[1,30]$. Like with BDBSs, cholangitis was another common adverse event of FCSMESs. FCSEMSs are usually designed with a larger diameter to a have longer patency period. However, this design also results in a reflux of duodenal contents, leading to cholangitis and sludge occlusion [42]. For MPSs, cholangitis resulting from frequent interventions was the most common adverse event.

To overcome the above limitations of these three stents, some newly designed stents and improved versions of current stents have emerged. For example, to prevent the reverse flow from duodenal lumen when using FCSMESs, an anti-reflux valve has been added at the duodenal end of these stents. As reported, this anti-reflux metal stent not only reduces the risk of ascending cholangitis during follow-up, but also prolongs the stent patency period [42]. To prevent the formation of biofilm on the inner surface of FCSEMSs, silver particles have been integrated in the silicone membrane. Because of the broad and effective antimicrobial activity of silver particles, the biofilm thicknesses on the surface of this stent was only $99.8 \mu \mathrm{m}$, which was dramatically reduced when compared with control group (122.9 $\mu \mathrm{m})$. In addition, the inhibiting effect of silver particles also reduced the sludge impaction in this stent, leading to a longer patency period than conventional silicone-covered stents (179 vs. 116.5 days) [43]. Recently, a new kind of biodegradable stent, which behaves similarly to standard plastic stents, was designed with a helicoidal shape to deal with biliary stricture. The bile could flow through the double-spiral channel existing in the outer shell and centre core of this stent, which might reduce the possibility of stent obstruction. In addition, because this kind of biodegradable stent can be effectively implanted using common devices, there is no concern about readjustment of the position of the stent with special equipment [41].

To date, there have been many articles discussing which stent is more appropriate for malignant biliary stricture; however, few articles have been written about BBS. It is worth noting that the conclusions drawn about malignant biliary stricture were not ap- plicable to BBS because of the significant differences in aetiology, prognosis, and survival time between these 2 conditions. Although a recent meta-analysis on BBS was published in 2020 by Almeida et al. [44], the authors only compared MPSs and BDBSs, not including FCSEMSs and the related articles from the last 2 years. On the other hand, they did not make necessary corrections in the statistical analysis of included data. For example, during the analysis of long-term stricture remission rates, the direct application of uncorrected data resulted in a $95 \% \mathrm{Cl}$ of more than $100 \%$ in 2 included papers, which was unreasonable. In the current meta-analysis, we used the statistical method of Freeman-Tukey transformation to correct the data because some of the incidences were close or equal to $100 \%$. In addition, compared with previously published meta-analyses, the outcome measures in our study were more comprehensive, thus providing a reference to select an appropriate stent.

This single-arm meta-analysis had several limitations worthy of mention. First, because most included studies were case series reports, the experience of operator, endoscopic insertion device, stent implantation method, and definitions of outcome measures differed widely, which led to the pooled results lacking credibility. Second, because the meta-analysis included a large number of retrospective studies, some of the authors might be biased in reporting effective cases, which may have inflated the probability of technical success, treatment success, and clinical success. Third, the small number of included studies for the BDBS group and the MPS group rendered the pooled data unconvincing. Fourth, although some causes of BBS, such as CP and LT, were reported to have poor prognosis, we did not subgroup these aetiologies in the current meta-analysis, leading to the limited reference value of this study.

\section{Conclusions}

Despite these limitations, our meta-analysis provides a better understanding of the application of 3 common stents in BBS. Although prospective and controlled trials in this regard were unavailable and considerable heterogeneity was identified, this study demonstrated that the pooled clinical success rate of BDBSs was superior to those of FCSMESs and MPSs. The technical success, treatment success, and 
adverse event rate were also better in BDBSs than the other 2 stents, although their stricture recurrence tended to be more common. The conclusions drawn from this meta-analysis should be further confirmed by well-designed RCTs with large samples and long-term follow-up.

\section{Acknowledgments}

This work was supported by the National Natural Science Foundation of China (Grant No. 82000485).

\section{Conflict of interest}

The authors declare no conflict of interest.

\section{References}

1. Coté GA, Slivka A, Tarnasky P, et al. Effect of covered metallic stents compared with plastic stents on benign biliary stricture resolution: a randomized clinical trial. JAMA 2016; 315: 1250-7.

2. Lawrence C, Romagnuolo J, Payne KM, et al. Low symptomatic premature stent occlusion of multiple plastic stents for benign biliary strictures: comparing standard and prolonged stent change intervals. Gastrointest Endosc 2010; 72: 558-63.

3. Saxena P, Diehl DL, Kumbhari V, et al. A US multicenter study of safety and efficacy of fully covered self-expandable metallic stents in benign extrahepatic biliary strictures. Dig Dis Sci 2015 60: 3442-8

4. Wong MY, Saxena P, Kaffes AJ. Benign biliary strictures: a systematic review on endoscopic treatment options. Diagnostics 2020; 10: 221.

5. Dopazo C, Diez I, Quintero J, et al. Role of biodegradable stents as part of treatment of biliary strictures after pediatric and adult liver transplantation: an observational single-center study. J Vasc Interv Radiol 2018; 29: 899-904.

6. Wu J, Zhou DX, Wang TT, et al. A new fully covered self-expandable metal stent for the treatment of postsurgical benign biliary strictures. Dig Dis Sci 2017; 62: 2550-7.

7. Kahaleh M, Brijbassie A, Sethi A, et al. Multicenter trial evalu ating the use of covered self-expanding metal stents in benign biliary strictures: time to revisit our therapeutic options? I Clin Gastroenterol 2013; 47: 695-9.

8. Costamagna G, Tringali A, Perri V, et al. Endotherapy of postcholecystectomy biliary strictures with multiple plastic stents: long-term results in a large cohort of patients. Gastrointest Endosc 2020; 91: 81-9.

9. Lakhtakia S, Reddy N, Dolak W, et al. Long-term outcomes after temporary placement of a self-expanding fully covered metal stent for benign biliary strictures secondary to chronic pancreatitis. Gastrointest Endosc 2020; 91: 361-9.

10. Aepli P, St John A, Gupta S, et al. Success and complications of an intra-ductal fully covered self-expanding metal stent (ID-FCSEMS) to treat anastomotic biliary strictures (AS) after orthotopic liver transplantation (OLT). Surg Endosc 2017; 31: 1558-63.
11. Wagh MS, Chavalitdhamrong D, Moezardalan K, et al. Effectiveness and safety of endoscopic treatment of benign biliary strictures using a new fully covered self-expandable metal stent. Diagn Ther Endosc 2013; 2013: 183513.

12. Hu B, Gao DJ, Yu FH, et al. Endoscopic stenting for post-transplant biliary stricture: usefulness of a novel removable covered metal stent. J Hepatobiliary Pancreat Sci 2011; 18: 640-5.

13. Javed A, Shashikiran BD, Aravinda PS, et al. Laparoscopic versus open surgery for the management of post-cholecystectomy benign biliary strictures. Surg Endosc 2021; 35: 1254-63.

14. Yang Q, Liu J, Ma W, et al. Efficacy of different endoscopic stents in the management of postoperative biliary strictures: a systematic review and meta-analysis. J Clin Gastroenterol 2019; 53: 418-26.

15. Siiki A, Rinta-Kiikka I, Sand J, et al. A pilot study of endoscopically inserted biodegradable biliary stents in the treatment of benign biliary strictures and cystic duct leaks. Gastrointest Endosc 2018; 87: 1132-7.

16. Lee JH. How far have we come in the design of biliary stents: are biodegradable stents ready for prime time? Gastrointest Endosc 2020; 92: 412-4

17. Higgins JL, Thomas J. Cochrane Handbook for Systematic Reviews of Interventions Version 6. The Cochrane Collaboration, 2019. Available from: https://training.cochrane.org/handbook/ current.

18. Wells G, Shea B, O'Connell D, et al. The Newcastle-Ottawa Scale (NOS) for assessing the quality of non-randomized studies in meta-analyses. Available from: http://www.ohri.ca/programs/ clinical epidemiology/oxford.asp 2001.

19. De Gregorio MA, Criado E, Guirola JA, et al. Absorbable stents for treatment of benign biliary strictures: long-term follow-up in the prospective Spanish registry. Eur Radiol 2020; 30: 448695.

20. Battistel M, Senzolo M, Ferrarese A, et al. Biodegradable biliary stents for percutaneous treatment of post-liver transplantation refractory benign biliary anastomotic strictures. Cardiovasc Intervent Radiol 2020; 43: 749-55.

21. Mauri G, Michelozzi C, Melchiorre F, et al. Benign biliary strictures refractory to standard bilioplasty treated using polydoxanone biodegradable biliary stents: retrospective multicentric data analysis on 107 patients. Eur Radiol 2016; 26: 4057-63.

22. Mauri G, Michelozzi C, Melchiorre F, et al. Treatment of benign biliary strictures with bioabsorbable biliary stent: preliminary results of a multicentric study. ECR 2015; B-1124.

23. Giménez ME, Palermo M, Houghton E, et al. Biodegradable biliary stents: a new approach for the management of hepaticojejunostomy strictures following bile duct injury. Prospective Study. Arq Bras Cir Dig 2016; 29: 112-6.

24. Sato T, Kogure H, Nakai Y, et al. A prospective study of fully covered metal stents for different types of refractory benign biliary strictures. Endoscopy 2020; 52: 368-76.

25. Poley JW, Ponchon T, Puespoek A, et al. Fully covered self-expanding metal stents for benign biliary stricture after orthotopic liver transplant: 5-year outcomes. Gastrointest Endosc 2020; 92: 1216-24

26. Tringali A, Reddy DN, Ponchon T, et al. Treatment of post-cholecystectomy biliary strictures with fully-covered self-expanding 
metal stents - results after 5 years of follow-up. BMC Gastroenterol 2019; 19: 214.

27. Schmidt A, Pickartz T, Lerch MM, et al. Effective treatment of benign biliary strictures with a removable, fully covered, self-expandable metal stent: a prospective, multicenter European study. United Eur Gastroenterol I 2017; 5: 398-407.

28. Chaput U, Vienne A, Audureau E, et al. Temporary placement of fully covered self-expandable metal stents for the treatment of benign biliary strictures. United European Gastroenterol J 2016; 4: 403-12.

29. Walter D, Laleman W, Jansen JM, et al. A fully covered self-expandable metal stent with antimigration features for benign biliary strictures: a prospective, multicenter cohort study. Gastrointest Endosc 2015; 81: 1197-203.

30. Hu B, Leung JW, Gao DJ, et al. Management of benign biliary strictures with a novel retrievable self-expandable metal stent. J Dig Dis 2014; 15: 146-53.

31. Ryu CH, Kim MH, Lee SS, et al. Temporary placement of fully covered self-expandable metal stents in benign biliary strictures. Korean J Gastroenterol 2013; 62: 49-54.

32. Poley JW, Cahen DL, Metselaar HJ, et al. A prospective group sequential study evaluating a new type of fully covered self-expandable metal stent for the treatment of benign biliary strictures (with video). Gastrointest Endosc 2012; 75: 783-9.

33. Moon JH, Choi HJ, Koo HC, et al. Feasibility of placing a modified fully covered self-expandable metal stent above the papilla to minimize stent-induced bile duct injury in patients with refractory benign biliary strictures (with videos). Gastrointest Endosc 2012; 75: 1080-5.

34. Park DH, Lee SS, Lee TH, et al. Anchoring flap versus flared end, fully covered self-expandable metal stents to prevent migration in patients with benign biliary strictures: a multicenter, prospective, comparative pilot study (with videos). Gastrointest Endosc 2011; 73: 64-70.

35. Ohyama H, Mikata R, Ishihara T, et al. Efficacy of multiple biliary stenting for refractory benign biliary strictures due to chronic calcifying pancreatitis. World I Gastrointest Endosc 2017; 9: 12-8.

36. Canena J, Liberato M, Coutinho AP, et al. Predictive value of cholangioscopy after endoscopic management of early postcholecystectomy bile duct strictures with an increasing number of plastic stents: a prospective study (with videos). Gastrointest Endosc 2014; 79: 279-88.

37. Bazerbachi F, Sawas T, Vargas EJ, et al. Metal stents versus plastic stents for the management of pancreatic walled-off necrosis: a systematic review and meta-analysis. Gastrointest Endosc 2018; 87: 30-42.

38. Haffar S, Bazerbachi F, Garg S, et al. Frequency and prognosis of acute pancreatitis associated with acute hepatitis E: a systematic review. Pancreatology 2015; 15: 321-6.

39. Siiki A, Helminen M, Sand J, et al. Covered self-expanding metal stents may be preferable to plastic stents in the treatment of chronic pancreatitis-related biliary strictures: a systematic review comparing 2 methods of stent therapy in benign biliary strictures. J Clin Gastroenterol 2014; 48: 635-43.

40. Niu F, Liu YD, Chen RX, et al. Safety and efficacy of enhanced recovery after surgery in elderly patients after therapeutic en- doscopic retrograde cholangiopancreatography. Videosurgery Miniinv 2019; 14: 394-400.

41. Anderloni A, Fugazza A, Maroni L, et al. New biliary and pancreatic biodegradable stent placement: a single-center, prospective, pilot study (with video). Gastrointest Endosc 2020; 92: 405-11.

42. Hamada T, Nakai Y, Isayama $\mathrm{H}$, et al. Antireflux metal stent for biliary obstruction: any benefits? Dig Endosc 2021; 33: 310-20.

43. Lee TH, Jung MK, Kim TK, et al. Safety and efficacy of a metal stent covered with a silicone membrane containing integrated silver particles in preventing biofilm and sludge formation in endoscopic drainage of malignant biliary obstruction: a phase 2 pilot study. Gastrointest Endosc 2019; 90: 663-72.

44. Almeida G, Donato P. Biodegradable versus multiple plastic stent implantation in benign biliary strictures: a systematic review and meta-analysis. Eur J Radiol 2020; 125: 108899.

Received: 7.05 .2021 , accepted: 20.06.2021. 